\title{
23. GEOCHEMISTRY AND ISOTOPIC DATING OF CENOZOIC VOLCANIC ARC SEQUENCES AROUND THE CELEBES AND SULU SEAS ${ }^{1}$
}

\author{
H. Bellon ${ }^{2}$ and C. Rangin ${ }^{3}$
}

\begin{abstract}
Geochemical data and whole-rock ${ }^{40} \mathrm{~K}-{ }^{40} \mathrm{Ar}$ isotopic ages are presented for more than 50 igneous rocks (a majority of lavas and some plutonic bodies) sampled onshore (Philippine Archipelago: Tablas, Panay, Masbate, Mindanao, northern Borneo (Sabah), and north Sulawesi) around the Celebes and Sulu Seas. These data are compared with the ${ }^{40} \mathrm{~K}-{ }^{40} \mathrm{Ar}$ ages obtained on drilled lavas along the Cagayan Ridge at ODP Sites 769 and 771 and with the major pyroclastic and tephras events recorded in the basins. Onshore ages range from $32 \mathrm{Ma}$ to near $0 \mathrm{Ma}$ for these rocks of generally calc-alkaline affinity with some shoshonitic high-K basalts. On the basis of geological data and kinematic reconstructions, two types of island arcs can be differentiated: those related to the progressive closing of the Celebes and Sulu marginal basins and those belonging to the Philippine Sea Plate. The combined age and chemistry for these two magmatic belts allow us to decipher the Neogene evolution of the complex zone of interaction of the Eurasian, Philippine Sea, and Australian plates.
\end{abstract}

\section{INTRODUCTION}

The Celebes and Sulu Seas marginal basins lie southeast of the South China Sea within the complex zone of junction of Philippine Sea, Indian, and Pacific plates. These two restricted northeast-trending basins are bounded (Fig. 1) along their eastern margins by the Philippine Archipelago, on their western edge by the island of Borneo, and on the south by the north arm of Sulawesi. From northwest to southeast, three ridges, the emerged Palawan Ridge, the submerged Cagayan Ridge, and Sulu Ridge are northeast-southwest trending. This last one is the locus of historical arc volcanism (Sulu Archipelago).

This paper has two main objectives: (1) A presentation of new data (isotopic ages and geochemical compositions) obtained from onshore magmatism around these marginal basins and (2) a comparison of these data and those obtained by similar methods from the offshore magmatism studied during Leg 124 along the Cagayan Ridge (Rangin, Silver, von Breymann, et al., 1990; Pubellier et al., this volume) and during previous dredging along this ridge (Kudrass et al., 1986 and 1989) to ascertain the timing of the onshore and offshore magmatism.

\section{METHODS AND DATA REDUCTION}

Forty-seven lavas and ten plutonic rocks collected onshore around the two basins, and five lavas drilled at Sites 769 and 771 during Leg 124 were dated by ${ }^{40} \mathrm{~K}-{ }^{40} \mathrm{Ar}$ method. Results are listed in Table 1. Forty-three geochemical analyses are reported in Table 2. Sampling locations, types of rocks, and resumed isotopic age relationships are given in Table 3.

A sketch map of the studied areas with the sample locations (Fig. 2) shows that the offshore sampling is representative of the various magmatic arc assemblages surrounding the Celebes and Sulu basins.

\footnotetext{
1 Silver, E. A., Rangin, C., von Breymann, M. T., et al., 1991. Proc. ODP. Sci. Results, 124: College Station, TX (Ocean Drilling Program).

${ }^{2}$ Laboratoire de Géochimie et de Géochronologie, URA 1278 CNRS et GDR 910, Université de Bretagne Occidentale, 29287 Brest Cedex, France.

${ }^{3}$ Laboratoire de Géologie Stucturale, URA 1315 CNRS T 26-01, Université Pierre et Marie Curie 4, place Jussieu, 75252 Paris Cedex, France.
}

The Cagayan Ridge can be traced to Panay Island and probably also to Tablas. The Sulu Ridge extends to Sabah and the Zamboanga Peninsula.

The north arm of Sulawesi is also the site of still-active volcanism in its eastern segment that started at least 5 m.y. ago. This segment can be extended northward to the Sangihe and Kawio islands. Several active volcanoes occur in the Sangihe Islands. Isotopic ages of $15.6,5.7$, and $2-0.9 \mathrm{Ma}$ are reported by Morrice et al. (1983) for the Kawio Islands. This activity is related to the final stage of subduction of the Molucca Plate (Silver et al., 1983, Moore et al., 1982). A new subduction zone was recently developed along the northern side of the north arm of Sulawesi, initiating subduction of the Celebes Basin floor toward the south (Silver et al., 1983).

Mindanao and Panay islands are mainly composed of Cainozoic island arc assemblages forming the backbone of the Philippine Mobile Belt (Gervaiso, 1971). This belt is composed of fragments of the Cagayan, Sulu, and north Sulawesi island arc terranes but mainly of exotic volcanic arc terranes interpreted as parts of the Philippine volcanic arc (Rangin and Pubellier, 1990). This arc was formerly attached to the Philippine Sea Plate before incipient subduction along the Philippine Trench occurred in early Pliocene time (Rangin et al., 1990a, Aurelio et al., 1990).

The Philippine Mobile Belt is not composed not only of the fragments of the Philippine Sea Plate but also of minor fragments of the Cagayan-Sulu and north Sulawesi island arcs (Rangin et al., 1990a).

\section{Isotopic Ages: Experimental Procedures and Age Calculations}

Analyses were performed on whole-rock samples (except one from Panay, for which separated biotites and whole rock were both analyzed). The $0.5-$ to $0.16-\mathrm{mm}$ size fraction was selected after crushing and sieving, and was carefully cleaned with distilled water. Potassium was determined by atomic absorption techniques from solutions of powders made from splits from this grain fraction.

Argon extraction from an average 1-g sample wrapped in an aluminum foil with an average weight of $0.125 \mathrm{~g}$ was performed by induction heating under high vacuum. Cleaning of active gases was achieved by a series of titanium furnaces 


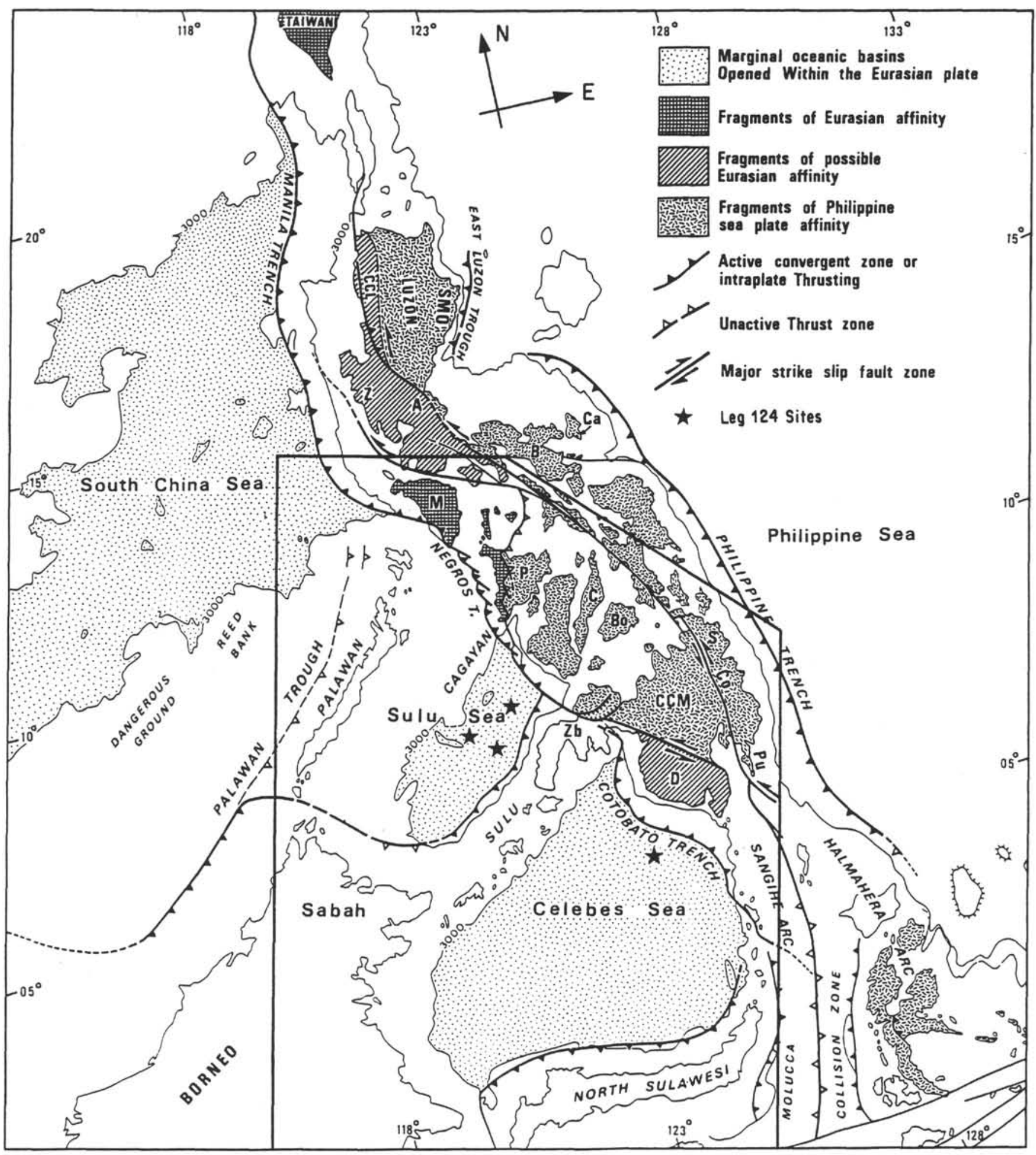

Figure 1. Simplified structural map of the Philippines and of the Sulu and Celebes oceanic basins opened within the Eurasian Plate. Suspected origins for the structural units of the Philippine Mobile Belt are distinguished: Eurasian- or Philippine Sea Plate affinities (after Rangin et al., 1990a). The study area is outlined. $\mathrm{P}=\mathrm{Panay}$, and in Mindanao: $\mathrm{S}=$ Surigao, $\mathrm{Co}=$ Eastern cordillera, $\mathrm{CCM}=$ central cordillera, $\mathrm{D}=\mathrm{Daguma}$ Range, $\mathrm{Zb}=$ Zamboanga. 
and a final gettering by two $\mathrm{Al}-\mathrm{Zr}$ getters. The argon isotopic composition was measured by mass spectrometry with a reference to air-argon composition measured in the same way after each sample to correct mass discrimination effects.

Radiogenic ${ }^{40} \mathrm{Ar}$ was determined by isotopic dilution using an original procedure described in (Bellon et al., 1981), where $\mathrm{a}^{38} \mathrm{Ar}$ spike is buried as ions in an aluminum foil target. Each target, with a precise ${ }^{38} \mathrm{Ar}$ volume regularly calibrated with standard samples (Glauconite Gl-O)(Cassignol et al., 1977) was added to the sample at the time of weighing. Consequently, isotope dilution was achieved during fusion of the sample.

Isotopic ages reported in Table 1 are calculated using the constants recommended by Steiger and Jäger (1977). Errors are estimates of the standard deviation of precision and are calculated using the extension of the Cox-Dalrymple (1967) error equation proposed by Mahood and Drake (1982). The extended equation includes an additional term (1\%) for the spectrometer mass discrimination. Analytical parameters $\left({ }^{40} \mathrm{Ar}_{\mathrm{R}}\right.$ (radiogenic); \% ${ }^{40} \mathrm{Ar}_{\mathrm{R}} ;{ }^{36} \mathrm{Ar}$ ) are only related to the sample, i.e. each value is corrected for nonradiogenic $\operatorname{Ar}\left({ }^{40} \mathrm{Ar}\right.$ and ${ }^{36} \mathrm{Ar}$ ) of atmospheric composition linked to the wrapping aluminum foil $\left({ }^{36} \mathrm{Ar}=3.5 \times 10^{-10} \mathrm{~cm}^{3}\right)$ and to the blank line that varies for a set of nine samples from ${ }^{36} \mathrm{Ar}=7 \times 10^{-10} \mathrm{~cm}^{3}$ (in the first extraction) to ${ }^{36} \mathrm{Ar}=1.2<2410^{-10} \mathrm{~cm}^{3}$ (in the ninth extraction). Repeated blank analyses were processed in the same way as for geologic samples, using only an aluminum foil target with buried ${ }^{38} \mathrm{Ar}$ ions.

\section{Geochemistry of Igneous Rocks}

Data from Table 2 are displayed on a $\mathrm{K}_{2} \mathrm{O}$ vs. $\mathrm{SiO}_{2}$ diagram (Fig. 3) with the superimposed fields of arc tholeiitic, calcalkaline, high-K calc-alkaline, and shoshonitic suites of Peccerillo and Taylor (1976), modified after Maury (1984). Note that in both diagrams ( $3 \mathrm{~A}$ and $3 \mathrm{~B}$ ), full symbols mark rocks younger than $8 \mathrm{Ma}$.

The lavas span a range from basalts to rhyodacites. In Figure $3 \mathrm{~A}$ are plotted dated lavas and plutonic rocks from western Panay, Tablas, and Sabah, and the drilled lavas along the Cagayan Ridge. Many of the rocks are located within the calc-alkaline field or slightly overlap its upper limit.

Onshore rocks from Eastern Panay, Mindanao, and Masbate are plotted in Figure 3B.

Rocks that plot within the arc tholeiitic field have been examined on the basis of their $\mathrm{FeO}_{2}{ }^{*} / \mathrm{MgO}_{(\mathrm{FeO}}{ }^{*}=$ total iron as $\mathrm{FeO}$ ). According to Gill (1981), arc tholeiitic rocks are lying above a line $\mathrm{FeO} * / \mathrm{MgO}=0.1562 \times \mathrm{SiO}_{2}-6.685$. Only a few analyzed rocks belong to an arc tholeiitic suite: a young basalt from Sabah (Sample S 129), a gabbro into a melange from Dent Peninsula (S 87-34) and a diorite (MNO 89-26A) from Daguma Range in Mindanao. Other rocks from this field belong to a low-K calc-alkaline suite.

Some shoshonitic basalts are associated in space and time with calc-alkaline suite as in western Panay (Fig. 3A), eastern Panay, and among the young volcanoes in Mindanao (Fig. 3B).

Figure 4 shows the normalized incompatible elements patterns for some selected basalts (4A) and andesites (4B). Large variations in $\mathrm{Rb}, \mathrm{Ba}, \mathrm{K}$, and $\mathrm{Sr}$ concentrations (from 10 to 300 times the chondritic values) are evidenced in Figure 4A. $\mathrm{P}$ and Ti element abundances also show a large range ( 8 to 70 times the chondritic values for phosphorus and 7 to 25 times for titanium).

Arc tholeiitic basalt from Sabah (Sample S 129) has a typical flat pattern, normalized values being 15 to 25 times the chondritic values.
Incompatible elements patterns for andesites span a narrow range in comparison with those for basalts; normalized abundances of $\mathrm{Rb}, \mathrm{Ba}$, and $\mathrm{K}$ are between 40 and 150 times the chondritic values; those of $\mathrm{Sr}, \mathrm{P}$, and $\mathrm{Ti}$ are respectively 25 to 60,15 to 35 , and 6 to 15 times the chondritic values. The patterns for $\mathrm{Rb}, \mathrm{Ba}$, and $\mathrm{K}$ are almost flat.

\section{PANAY}

\section{General Geological Overviews}

Panay Island (Fig. 5) belongs to the Philippine Mobile Belt and to the Negros arc. In western Panay, the Antique Range is formed by northeast-elongated island arc terranes with ophiolites and melanges, which are thrusting over the Cuyo shelf. This arc outcrops in the Buruanga Peninsula. The Antique Range is the northernmost extension of the Negros Trench inner wall. The Iloilo Basin onlaps westward on the Antique Range uppermost unit and eastward on the Eastern Range.

A detailed stratigraphic and structural study of the different units completed by isotopic dates of volcanics (Rangin et al., in press) shows that this sequence has formed by the collision of several Tertiary volcanic arcs with the North Palawan block, a continental rifted fragment of the Eurasian continental margin.

\section{Field Identification of the Arc Units}

From north to south, the Antique Range is composed of:

1. The unit at the northwestern tip of the Buruanga Peninsula, that is made of a mainly pre-Tertiary metamorphic basement intruded by later plutonic bodies (Patria and east of Pandan);

2. A northeast-trending, mainly volcanic terrane, the Baloy unit extending from Naba to Cubay River formed by volcanic rocks overlain by a detrital formation with interlayered basalts (Mayos Fm);

3. Another northeast-trending volcanic terrane, the Valderrama unit extending from Cubay River to Pampanan thrust was subdivided in two series: a volcanic and volcano-sedimentary one (Lagdo Fm) and its basement made of Cretaceous ophiolites (Lombonero Ridge) and a late Paleogene melange (Panicuan).

The Iloilo unit consists of a clastic basinal sequence (Iloilo Basin) that rests on a volcanic basement. This basement is exposed east and west of this north-south-trending elongated undeformed basin. On its eastern edge, the Iloilo Basin rests on the Eastern Range formations including volcanic and sedimentary sequences intruded by plutonic bodies (Sara diorite). Along its western edge, in the Antique Range, sediments are conformably deposited on volcanic rocks thrusted westward on the Valderrama unit. The basin ends as a "cul de sac" in the Dumalog area (Fig. 5) in central Panay.

\section{Isotopic Ages}

Fifteen selected samples (Tables 1 and 3) for ${ }^{40} \mathrm{~K}-{ }^{40} \mathrm{Ar}$ whole-rock dating yield ages ranging between 30 and $12.4 \mathrm{Ma}$. According to their field locations (Fig. 5), the data may be separated into three groups.

The oldest dates ( 30 to $21.5 \mathrm{Ma}$ ) were obtained on the volcanic rocks from the Iloilo Basin basement. This includes lavas from the Dumalog area that were all dated at 30 to $26 \mathrm{Ma}$. Ages from volcanics collected along the western flank of the basin are constrained by biostratigraphic data; in the Tipuluan River up to a thousand meters of volcanic breccias with intercalated flows and argilitic layers were dated lower Miocene. Between San Jose and Tigmarabo, several hundred meters of agglomerates and flows cap $1200 \mathrm{~m}$ of graywackes, siltstones, 
Table 1. Conventional ${ }^{40} \mathrm{~K}-{ }^{40} \mathrm{Ar}$ Age Data on Samples from onshore magmatism (Panay, Tablas, Sabah, Mindanao, Masbate) and offshore volcanism (Cagayan Ridge, Leg 124, Sites 769 and 771).

\begin{tabular}{|c|c|c|c|c|c|c|}
\hline $\begin{array}{c}\text { (a) } \\
\text { Sample }\end{array}$ & $\begin{array}{c}\text { (b) } \\
\text { Calculated Age } \\
\pm \text { error }\end{array}$ & $\begin{array}{c}(\mathrm{c} \text { and d) } \\
40 \mathrm{Ar} \mathrm{R}(\mathrm{a}) \\
\left(10^{-7} \mathrm{~cm}^{3} / \mathrm{g}\right)\end{array}$ & $\begin{array}{c}{ }_{36}^{(\mathrm{c})} \\
\left(10^{-9} \mathrm{~cm}^{3} / \mathrm{g}\right)\end{array}$ & $\%^{(d)}$ Ar R & $\begin{array}{r}\mathrm{K}_{2} \mathrm{O} \\
(\%)\end{array}$ & $\begin{array}{l}\text { Analysis } \\
\text { Number }\end{array}$ \\
\hline \multicolumn{7}{|c|}{ PANAY } \\
\hline \multicolumn{7}{|c|}{ Valderrama } \\
\hline P 83-57 & $13.11 \pm 0.91$ & 13.19 & 11.58 & 27.8 & 3.11 & B $588-3$ \\
\hline P 83-91 & $12.40 \pm 0.84$ & 3.49 & 2.99 & 28.4 & 0.87 & B 721-6 \\
\hline P 83-93 & $13.34 \pm 0.76$ & 3.24 & 2.25 & 32.7 & 0.75 & B2158-7 \\
\hline P $83-94$ & $13.72 \pm 0.82$ & 8.48 & 6.28 & 29.2 & 1.91 & B2131-2 \\
\hline P 83-97 & $13.83 \pm 1.16$ & 5.86 & 6.34 & 23.8 & 1.31 & B2308-1 \\
\hline \multicolumn{7}{|c|}{ Baloy Mount } \\
\hline P 87-39a & $14.98 \pm 0.52$ & 4.61 & 1.69 & 47.9 & 0.95 & B2157-6 \\
\hline P 87-43a & $22.87 \pm 0.75$ & 8.90 & 2.95 & 50.6 & 1.20 & B2062-5 \\
\hline \multicolumn{7}{|c|}{ Buruanga } \\
\hline P $83-114$ (RT)* & $19.49 \pm 0.47$ & 17.75 & 3.26 & 64.8 & 2.81 & B $578-2$ \\
\hline P 83-114 (B) & $20.80 \pm 0.61$ & 52.41 & 14.24 & 55.4 & 7.77 & B 577-1 \\
\hline P $83-117^{*}$ & $20.85 \pm 0.87$ & 11.02 & 5.17 & 41.9 & 1.63 & B $586-1$ \\
\hline \multicolumn{7}{|c|}{ Iloilo Basement } \\
\hline P $83-47 e$ & $21.51 \pm 0.55$ & 14.65 & 4.12 & 54.6 & 2.10 & B2121-1 \\
\hline P 83-29 & $23.67 \pm 1.33$ & 6.14 & 4.21 & 33.1 & 0.80 & B2340-3 \\
\hline P $83-46$ & $25.35 \pm 0.70$ & 12.02 & 2.94 & 58.1 & 1.46 & B $720-5$ \\
\hline P 83-19 & $26.20 \pm 0.54$ & 28.50 & 3.08 & 75.8 & 3.35 & B2338-1 \\
\hline & $25.75 \pm 0.51$ & 28.01 & 2.44 & 79.6 & 3.35 & B2334-3 \\
\hline P 83-22a & $30.96 \pm 0.57$ & 34.63 & 1.54 & 88.3 & 3.44 & B2339-2 \\
\hline & $29.20 \pm 0.52$ & 32.64 & 0.99 & 91.8 & 3.44 & B2354-1 \\
\hline \multicolumn{7}{|c|}{ Eastern Range } \\
\hline P 87-67b* & $29.69 \pm 0.58$ & 16.89 & 1.29 & 81.6 & 1.75 & B2061-4 \\
\hline \multicolumn{7}{|c|}{ TABLAS } \\
\hline TA 31 & $3.78 \pm 0.28$ & 1.10 & 1.05 & 26.2 & 0.90 & B1804-3 \\
\hline TA 32 & $4.39 \pm 0.47$ & 1.43 & 2.01 & 19.4 & 1.01 & B1805-4 \\
\hline TA 130 & $18.01 \pm 0.41$ & 6.54 & 1.04 & 68.0 & 1.12 & B1807-6 \\
\hline TA 43 & $18.48 \pm 0.36$ & 7.24 & 0.52 & 82.4 & 1.21 & B1806-5 \\
\hline TA 134 & $19.86 \pm 0.50$ & 8.30 & 1.70 & 62.4 & 1.29 & B1808-7 \\
\hline \multicolumn{7}{|c|}{ SABAH } \\
\hline \multicolumn{7}{|c|}{ Young Volcanics } \\
\hline S SUDE & $0.01 \pm 0.40$ & 0.00 & 0.62 & 0.0 & 0.37 & B1515-6 \\
\hline & $0.06 \pm 0.40$ & 0.01 & 0.81 & 0.3 & 0.37 & B2457-5 \\
\hline S 129 & $2.79 \pm 0.58$ & 0.27 & 0.76 & 10.7 & 0.30 & B1619-4 \\
\hline & $3.11 \pm 1.11$ & 0.30 & 1.47 & 6.5 & 0.30 & B1514-5 \\
\hline S $87-92$ & $2.94 \pm 1.30$ & 1.50 & 9.05 & 5.2 & 1.58 & B2205-2 \\
\hline & $3.29 \pm 1.06$ & 1.68 & 7.38 & 7.0 & 1.58 & B2066-9 \\
\hline \multicolumn{7}{|c|}{ Outer Belt : Kinabalu Intrusives } \\
\hline S $84-11=$ & $6.43 \pm 0.30$ & 6.67 & 3.63 & 38.4 & 3.21 & B $679-2$ \\
\hline S $84-16^{*}$ & $6.84 \pm 0.26$ & 8.90 & 3.72 & 44.7 & 4.03 & B $680-3$ \\
\hline \multicolumn{7}{|c|}{ Inner Belt: Dent Peninsula } \\
\hline S 87-34 & $11.47 \pm 0.81$ & 1.78 & 1.58 & 27.6 & 0.48 & B2064-7 \\
\hline S 264-1 & $11.53 \pm 0.47$ & 5.56 & 2.52 & 42.6 & 1.49 & B2196-2 \\
\hline S 264-3 & $12.58 \pm 0.33$ & 10.42 & 2.35 & 60.0 & 2.56 & B $1480-2$ \\
\hline S 267 & $11.07 \pm 0.33$ & 6.59 & 1.85 & 54.6 & 1.84 & B1477-8 \\
\hline S 266 & $12.69 \pm 0.56$ & 8.66 & 4.36 & 40.1 & 2.11 & B2458-6 \\
\hline S 268 & $11.69 \pm 0.37$ & 8.77 & 2.80 & 51.5 & 2.32 & B1495-5 \\
\hline \multicolumn{7}{|c|}{ Inner Belt : Semporna Peninsula } \\
\hline S $87-91$ & $9.01 \pm 0.21$ & 6.73 & 1.09 & 67.6 & 2.31 & B2065-8 \\
\hline S $87-90$ & $11.61 \pm 0.58$ & 9.50 & 4.21 & 43.2 & 2.53 & B2204-1 \\
\hline S $285-2$ & $11.80 \pm 0.59$ & 6.71 & 1.46 & 60.9 & 1.76 & B1509-5 \\
\hline S $138-1$ & $12.92 \pm 0.65$ & 6.94 & 1.14 & 67.3 & 1.66 & B1503-7 \\
\hline \multicolumn{7}{|c|}{ MINDANAO } \\
\hline \multicolumn{7}{|c|}{ Recent Volcanoes } \\
\hline MNO $89-16 B$ & $0.25 \pm 0.07$ & 0.12 & 0.43 & 5.6 & 1.45 & B2252-6 \\
\hline MNO 16A & $0.40 \pm 0.05$ & 0.41 & 0.67 & 15.9 & 3.21 & B2251-5 \\
\hline MNO 37 & $0.62 \pm 0.06$ & 0.37 & 0.47 & 19.3 & 1.86 & B2253-7 \\
\hline MNO 89-17 & $1.15 \pm 0.27$ & 0.37 & 1.19 & 8.5 & 1.00 & B2254-8 \\
\hline
\end{tabular}


Table 1 (continued).

\begin{tabular}{|c|c|c|c|c|c|c|}
\hline $\begin{array}{c}\text { (a) } \\
\text { Sample }\end{array}$ & $\begin{array}{c}\text { (b) } \\
\text { Calculated Age } \\
\pm \text { error }\end{array}$ & $\begin{array}{c}\text { (c and d) } \\
{ }^{40} \mathrm{Ar} \mathrm{R} \mathrm{(a)} \\
\left(10^{-7} \mathrm{~cm}^{3} / \mathrm{g}\right)\end{array}$ & $\begin{array}{c}(\mathrm{c}) \\
{ }^{36} \mathrm{Ar} \\
\left(10^{-9} \mathrm{~cm}^{3} / \mathrm{g}\right)\end{array}$ & $\%^{40} \mathrm{Ar} R$ & $\begin{array}{r}\mathrm{K}_{2} \mathrm{O} \\
(\%)\end{array}$ & $\begin{array}{l}\text { Analysis } \\
\text { Number }\end{array}$ \\
\hline \multicolumn{7}{|c|}{ Surigao del Sur } \\
\hline MNO 88-46 & $2.31 \pm 0.24$ & 2.07 & 2.84 & 19.7 & 2.77 & B2077-2 \\
\hline MNO 88-49 & $4.54 \pm 0.57$ & 1.92 & 3.22 & 16.8 & 1.31 & B2069-3 \\
\hline MNO 88-54 & $17.16 \pm 0.36$ & 7.34 & 0.91 & 73 & 1.32 & B2292-1 \\
\hline \multicolumn{7}{|c|}{ South Davao } \\
\hline MNO 89-21A & $9.31 \pm 0.18$ & 10.44 & 0.65 & 84.3 & 3.47 & B2258-3 \\
\hline MNO 89-21B & $10.64 \pm 0.22$ & 8.15 & 0.94 & 74.4 & 2.37 & B2249-3 \\
\hline \multicolumn{7}{|c|}{ Central Cordillera } \\
\hline MNO 89-12 & $16.32 \pm 0.85$ & 3.49 & 2.17 & 35 & 0.66 & B2468-7 \\
\hline MNO $89-20$ & $19.86 \pm 0.36$ & 0.36 & 0.46 & 92.1 & 2.46 & B2248-2 \\
\hline \multicolumn{7}{|c|}{ Daguma Range } \\
\hline MNO $89-25$ & $16.73 \pm 1.25$ & 3.03 & 2.88 & 26 & 0.56 & B2250-4 \\
\hline MNO 89-26A * & $29.89 \pm 2.12$ & 6.80 & 6.10 & 27.4 & 0.70 & B2213-1 \\
\hline MNO $89-26 B^{*}$ & $31.91 \pm 3.95$ & 2.02 & 3.31 & 17.1 & 0.19 & B2214-2 \\
\hline \multicolumn{7}{|c|}{ MASBATE } \\
\hline MAS 88-69 & $9.72 \pm 0.35$ & 12.13 & 4.71 & 46.6 & 3.86 & B2067-1 \\
\hline MAS $88-79 a$ & $10.64 \pm 0.56$ & 7.47 & 4.76 & 34.7 & 2.17 & B2161-1 \\
\hline \multicolumn{7}{|c|}{ NORTH SULAWESI : GORONTALO AREA } \\
\hline SN 89-01 & $4.10 \pm 0.31$ & 1.35 & 1.32 & 25.2 & 1.02 & B2393-7 \\
\hline SN 89-32 & $4.38 \pm 0.15$ & 1.85 & 0.67 & 47.8 & 1.31 & B2379-1 \\
\hline \multirow{2}{*}{ SN $89-47$} & $6.55 \pm 0.16$ & 1.31 & 0.27 & 62 & 0.62 & B2460-8 \\
\hline & $7.21 \pm 0.16$ & 1.44 & 0.21 & 69.7 & 0.62 & B2324-2 \\
\hline \multirow[t]{2}{*}{ SN 89-33* } & $8.79 \pm 0.19$ & 7.27 & 0.94 & 72.3 & 2.56 & B2453-1 \\
\hline & $8.88 \pm 0.17$ & 7.35 & 0.60 & 80.5 & 2.56 & B2387-1 \\
\hline \multirow{2}{*}{ SN $89-46^{*}$} & $18.23 \pm 0.42$ & 7.27 & 1.17 & 58.0 & 1.23 & B2454-2 \\
\hline & $18.72 \pm 0.44$ & 7.46 & 1.27 & 56.6 & 1.23 & B2388-2 \\
\hline SN $89-12 *$ & $22.24 \pm 0.46$ & 18.75 & 2.09 & 67.7 & 2.60 & B2370-1 \\
\hline \multicolumn{7}{|c|}{ SULU SEA : CAGAYAN RIDGE } \\
\hline \multicolumn{7}{|c|}{ Site 769} \\
\hline \multirow[t]{2}{*}{$7 \mathrm{R}-1,118-120 \mathrm{~cm}$} & $20.29 \pm 0.81$ & 7.70 & 9.45 & 42.9 & 1.17 & B2153-2 \\
\hline & $20.83 \pm 0.72$ & 7.90 & 2.84 & 48.3 & 1.17 & B2183-5 \\
\hline \multirow[t]{2}{*}{$9 \mathrm{R}-3,110-113 \mathrm{~cm}$} & $19.48 \pm 0.52$ & 10.54 & 2.47 & 59 & 1.67 & B2188-1 \\
\hline & $20.14 \pm 0.48$ & 10.90 & 1.89 & 66.1 & 1.67 & B2162-2 \\
\hline \multirow[t]{2}{*}{$9 \mathrm{R}-4,49-50 \mathrm{~cm}$} & $14.25 \pm 0.79$ & 8.02 & 5.43 & 33.2 & 1.74 & B2171-2 \\
\hline & $15.07 \pm 0.93$ & 8.49 & 6.51 & 30.5 & 1.74 & B2180-2 \\
\hline \multicolumn{7}{|c|}{ Site 771} \\
\hline $11 \mathrm{R}-2,91-94 \mathrm{~cm}$ & $18.78 \pm 2.38$ & 1.34 & 2.25 & 16.3 & 0.22 & B2174-5 \\
\hline & $20.90 \pm 2.90$ & 1.49 & 2.75 & 15.4 & 0.22 & B2189-2 \\
\hline \multirow[t]{2}{*}{$18 \mathrm{R}-7,142-144 \mathrm{~cm}$} & $14.23 \pm 0.34$ & 2.74 & 0.50 & 64.3 & 0.60 & B2476-2 \\
\hline & $13.84 \pm 0.39$ & 2.67 & 0.70 & 55.8 & 0.60 & B2477-3 \\
\hline
\end{tabular}

a: Asterisk that follows the sample code refers to its plutonic origin. b: Ages are calculated using constants recommended by Steiger and Jŭger (1977).c: Calculated concentrations only refer to sample. Blank values are deduced (see text for explanations). d: Subscript $\mathrm{R}$ means radiogenic ${ }^{40} \mathrm{Ar}$.

tuffs and conglomerates with calcareous levels bearing Foraminifers lenses of upper Oligocene to late lower Miocene. Underlying volcanic rocks range in age from 25.3 to $21.5 \mathrm{Ma}$. Lavas within the Eastern Range are probably older than $30 \mathrm{Ma}$ as they are intruded by dioritic bodies (Sara) dated 29.7 Ma.

The second set of ages, which ranges from 23 to $15 \mathrm{Ma}$, includes:

1. The intrusive of the Buruanga unit (Patria and Timbaban River near Pandan). Ages are from 20.9 to $19.5 \mathrm{Ma}$, comparable with the age $(20.8 \mathrm{Ma})$ of Tapian Mine tonalite on Marinduque island (Walther et al., 1981);

2. Volcanics of the Mt. Baloy unit were collected into two distinct thrust slices: one from the Tibiao River (22.9 Ma), and the other younger (15 Ma) in the Dalanas River.

3. The third group of ages (13.9 to $12.4 \mathrm{Ma}$ ) concerns volcanics from the Valderrama unit: 1. In the Cangaranan River, $5 \mathrm{~km}$ upstream of Valderrama; 2 . In the Inyaman River, that grooves Kipott Mount, made of volcanic breccias overlying limestones containing Zone NN9 nannofossils.

\section{Geochemical Compositions}

Several dated samples have been analyzed for their major elements and some trace elements (Table 2). Except three basalts (two from Iloilo Basin and one from the Valderrama volcanic sequence), the lavas are andesitic and plot in a $\mathrm{K}_{2} \mathrm{O}$ vs. $\mathrm{SiO}_{2}$ diagram (Figs. $3 \mathrm{~A}$ and $3 \mathrm{~B}$ ) in the medium-K calcalkaline field. Plutonic bodies of dioritic to granodioritic composition fall in the lower part of the high-K calc-alkaline field as does the Marinduque tonalite. All the rocks have a typical orogenic parentage.

Basalts are of special interest because they plot in the shoshonitic field. They have $\mathrm{K}_{2} \mathrm{O} / \mathrm{Na}_{2} \mathrm{O}$ ratios ranging from 1.6 to 3 and high $\mathrm{Cr}$ contents (150-230 ppm). Those from Iloilo basement have low $\mathrm{TiO}_{2}$ contents $(0.67 \%)$ and high $\mathrm{Ba}$ contents $(>1000 \mathrm{ppm})$ and can be tentatively related to the 
Table 2. Major- and trace-element concentrations of $\mathbf{4 3}$ samples from onshore magmatism and $\mathbf{4}$ offshore lavas drilled at Sites 769 and 771 during Leg 124. Analyses were performed at UBO by J. Cotten, analyst, using atomic absorption. Geochemical data are presented following the different geographical origins of sampling. LOI $=$ Loss on ignition.

\begin{tabular}{|c|c|c|c|c|c|c|c|c|c|c|c|c|}
\hline \multicolumn{13}{|c|}{ PANAY } \\
\hline \multirow{3}{*}{$\begin{array}{c}\text { (a) } \\
\text { Sample }\end{array}$} & \multirow{2}{*}{\multicolumn{3}{|c|}{-Valderrama $-\frac{}{15}$}} & \multirow{2}{*}{\multicolumn{2}{|c|}{ Baloy }} & \multirow{2}{*}{$\longrightarrow$} & \multicolumn{2}{|c|}{ Buruanga } & \multicolumn{2}{|r|}{-Iloilo } & 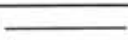 & \multirow{3}{*}{$\begin{array}{c}\text { E.Range } \\
1 \\
\mathrm{P}\end{array}$} \\
\hline & & & 15 & & & & 8 & 7 & 2 & 6 & 5 & \\
\hline & $\mathrm{P}$ & $\mathbf{P}$ & $\mathrm{P}$ & $\mathrm{P}$ & $\mathrm{P}$ & $\mathrm{P}$ & $\mathrm{P}$ & $P$ & $\mathrm{P}$ & $\mathbf{P}$ & $P$ & \\
\hline$(w t \%)$ & $83-91$ & $83-92$ & $83-57$ & $87-39 A$ & $87-43 \mathrm{~A}$ & $87-48 \mathrm{~A}$ & $83-114^{*}$ & $83-117^{*}$ & 83- 29 & $83-19$ & $83-22 \mathrm{~A}$ & 87-67B \\
\hline $\mathrm{SiO}_{2}$ & 54.90 & 61.50 & 50.00 & 53.00 & 54.80 & 47.20 & 64.55 & 59.30 & 58.00 & 50.05 & 49.50 & 55.50 \\
\hline $\mathrm{TiO}_{2}$ & 0.61 & 0.61 & 1.27 & 0.66 & 0.68 & 1.70 & 0.60 & 0.58 & 0.98 & 0.66 & 0.61 & 0.64 \\
\hline $\mathrm{Al}_{2} \mathrm{O}_{3}$ & 17.36 & 15.62 & 19.57 & 18.08 & 17.80 & 15.88 & 15.96 & 17.09 & 16.62 & 12.83 & 12.55 & 17.37 \\
\hline $\mathrm{Fe}_{2} \mathrm{O}_{3}$ & 8.35 & 4.03 & 7.23 & 7.08 & 7.34 & 11.30 & 4.84 & 5.92 & 7.89 & 10.32 & 10.10 & 8.33 \\
\hline $\mathrm{MnO}^{\circ}$ & 0.17 & 0.12 & 0.12 & 0.16 & 0.13 & 0.17 & 0.09 & 0.10 & 0.16 & 0.17 & 0.20 & 0.16 \\
\hline $\mathrm{MgO}$ & 3.89 & 0.87 & 4.83 & 5.75 & 3.55 & 8.10 & 2.38 & 3.37 & 3.30 & 7.59 & 7.96 & 3.74 \\
\hline $\mathrm{CaO}$ & 8.64 & 7.50 & 9.43 & 8.80 & 8.42 & 9.03 & 4.05 & 6.86 & 7.34 & 10.07 & 10.63 & 7.91 \\
\hline $\mathrm{Na}_{2} \mathrm{O}$ & 2.78 & 3.08 & 2.83 & 2.20 & 2.92 & 2.89 & 3.21 & 3.70 & 3.35 & 1.96 & 1.54 & 3.00 \\
\hline $\mathrm{K}_{2} \mathrm{O}$ & 0.89 & 2.49 & 2.65 & 1.04 & 1.20 & 0.94 & 2.81 & 1.65 & 0.79 & 3.15 & 3.61 & 1.75 \\
\hline $\mathrm{P}_{2} \mathrm{O}_{5}$ & 0.35 & 0.35 & 0.40 & 0.15 & 0.15 & 0.35 & 0.40 & 0.35 & 0.30 & 0.45 & 0.50 & 0.20 \\
\hline TOTAL & 99.74 & 99.39 & 99.25 & 99.59 & 100.17 & 100.02 & 99.68 & 99.38 & 99.94 & 100.02 & 99.48 & 99.96 \\
\hline $\begin{array}{l}\text { LOI } \\
\text { (ppm) }\end{array}$ & 1.81 & 3.22 & 0.92 & 3.80 & 3.28 & 2.46 & 0.79 & 1.06 & 1.21 & 2.77 & 2.28 & 1.36 \\
\hline $\mathrm{Sr}$ & 638 & 260 & 751 & 329 & 443 & 307 & 309 & 521 & 259 & 751 & 1605 & 597 \\
\hline $\mathrm{Rb}$ & 18 & 61 & 95 & 11 & 15 & 22 & 100 & 34 & 27 & 67 & 81 & 34 \\
\hline $\mathrm{Ba}$ & 158 & 255 & 333 & 195 & 275 & 245 & 417 & 194 & 136 & 1248 & 1012 & 472 \\
\hline $\mathrm{Ni}$ & 40 & 15 & 15 & 54 & 10 & 170 & 15 & 15 & 25 & 53 & 46 & 20 \\
\hline $\mathrm{Cr}$ & 27 & 50 & 23 & 44 & 13 & 230 & 50 & 48 & 30 & 233 & 148 & 6 \\
\hline V & 238 & 165 & 368 & 275 & 190 & 200 & 117 & 205 & 206 & 300 & 271 & 240 \\
\hline
\end{tabular}

SABAH - MASBATE

\begin{tabular}{|c|c|c|c|c|c|c|c|c|c|c|c|c|}
\hline & \multicolumn{2}{|c|}{ Young Volc } & \multirow{2}{*}{$\begin{array}{c}\text { Kinabalu } \\
11\end{array}$} & \multicolumn{4}{|c|}{-Dent } & \multicolumn{3}{|c|}{ Semporna } & \multicolumn{2}{|c|}{-Masbate - } \\
\hline & 13 & 12 & & 10 & 9 & 5 & 6 & 4 & 3 & 1 & 2 & 1 \\
\hline & $\mathbf{S}$ & $\mathbf{S}$ & S & $\mathbf{S}$ & $\mathrm{S}$ & $\mathbf{S}$ & $\mathrm{S}$ & $\mathrm{S}$ & $\mathrm{S}$ & $\mathrm{S}$ & MAS & MAS \\
\hline & & & $84-$ & & 264- & & & & & 138- & & 88- \\
\hline \multicolumn{13}{|l|}{ (wt\%) } \\
\hline $\mathrm{SiO}_{2}$ & 58.40 & 52.00 & 60.20 & 48.50 & 53.10 & 62.20 & 62.20 & 62.35 & 64.30 & 59.50 & 61.35 & 54.00 \\
\hline $\mathrm{TiO}_{2}$ & 1.06 & 2.27 & 0.64 & 0.69 & 0.98 & 0.57 & 0.64 & 0.50 & 0.54 & 0.77 & 0.82 & 0.94 \\
\hline $\mathrm{Al}_{2} \mathrm{O}_{3}$ & 16.19 & 14.12 & 16.65 & 18.92 & 17.58 & 15.85 & 16.69 & 15.98 & 15.53 & 16.00 & 16.35 & 18.44 \\
\hline $\mathrm{Fe}_{2} \mathrm{O}_{3}$ & 7.56 & 13.10 & 5.92 & 8.06 & 8.26 & 5.31 & 5.44 & 5.88 & 5.26 & 6.66 & 3.35 & 8.98 \\
\hline $\mathrm{MnO}$ & 0.15 & 0.15 & 0.14 & 0.17 & 0.14 & 0.10 & 0.08 & 0.11 & 0.10 & 0.14 & 0.20 & 0.18 \\
\hline $\mathrm{MgO}$ & 3.39 & 6.07 & 2.39 & 5.32 & 3.75 & 2.64 & 1.92 & 2.46 & 2.23 & 2.95 & 1.01 & 1.98 \\
\hline $\mathrm{CaO}$ & 4.47 & 6.60 & 5.51 & 10.03 & 8.92 & 5.13 & 6.51 & 6.00 & 4.84 & 6.05 & 3.42 & 7.21 \\
\hline $\mathrm{Na}_{2} \mathrm{O}$ & 3.48 & 3.06 & 2.98 & 2.92 & 2.54 & 3.21 & 3.01 & 2.73 & 3.06 & 2.89 & 4.26 & 3.78 \\
\hline $\mathrm{K}_{2} \mathrm{O}$ & 1.58 & 0.25 & 2.85 & 0.48 & 1.52 & 2.30 & 2.11 & 2.31 & 2.53 & 1.66 & 3.86 & 2.17 \\
\hline $\mathrm{P}_{2} \mathrm{O}_{5}$ & 0.25 & 0.15 & 0.20 & 0.05 & 0.30 & 0.20 & 0.25 & 0.12 & 0.10 & 0.10 & 0.30 & 0.35 \\
\hline TOTAL & 99.22 & 98.57 & 99.17 & 100.05 & 99.20 & 99.95 & 99.72 & 100.16 & 100.23 & 98.63 & 100.07 & 99.34 \\
\hline $\begin{array}{l}\text { LOI } \\
(\mathrm{ppm})\end{array}$ & 0.69 & 0.80 & 1.69 & 4.91 & 2.11 & 2.44 & 0.47 & 1.72 & 1.74 & 1.91 & 2.15 & 1.31 \\
\hline $\mathrm{Sr}$ & 350 & 162 & 466 & 326 & 457 & 482 & 423 & 270 & 245 & 210 & 436 & 500 \\
\hline $\mathrm{Rb}$ & 55 & 9 & 12 & 6 & 61 & 100 & 84 & 81 & 90 & 56 & 73 & 39 \\
\hline $\mathrm{Ba}$ & 371 & 77 & 409 & 71 & 335 & 439 & 365 & 312 & 349 & 430 & 760 & 460 \\
\hline $\mathrm{Ni}$ & 40 & 150 & 8 & 16 & 19 & 19 & 17 & 8 & 8 & 9 & 4 & 6 \\
\hline $\mathrm{Cr}$ & 68 & 232 & 13 & 33 & 10 & 30 & 24 & 10 & 14 & 3 & 3 & 10 \\
\hline V & 80 & 167 & 153 & 280 & 271 & 121 & 167 & 140 & 120 & 182 & 110 & 250 \\
\hline
\end{tabular}


Table 2 (continued).

TABLAS

\begin{tabular}{|c|c|c|c|c|c|}
\hline & ung arc & & & -Old arc & \\
\hline (a) & 5 & 4 & 3 & 2 & 1 \\
\hline Sample & TA & TA & TA & $\mathrm{TA}$ & TA \\
\hline & 31 & 32 & 130 & 43 & 134 \\
\hline (wt \%) & & & & & \\
\hline $\mathrm{SiO}_{2}$ & 60.90 & 65.50 & 60.00 & 61.35 & 69.50 \\
\hline $\mathrm{TiO}_{2}$ & 0.54 & 0.49 & 0.49 & 0.57 & 0.43 \\
\hline $\mathrm{Al}_{2} \mathrm{O}_{3}$ & 15.77 & 15.93 & 17.46 & 16.08 & 13.75 \\
\hline $\mathrm{Fe}_{2} \mathrm{O}_{3}$ & 5.02 & 4.36 & 5.17 & 5.56 & 3.35 \\
\hline $\mathrm{MnO}^{\circ}$ & 0.11 & 0.10 & 0.09 & 0.14 & 0.08 \\
\hline $\mathrm{MgO}$ & 3.97 & 2.79 & 3.43 & 3.31 & 0.75 \\
\hline $\mathrm{CaO}$ & 5.58 & 4.72 & 5.67 & 5.50 & 4.10 \\
\hline $\mathrm{Na}_{2} \mathrm{O}$ & 3.95 & 4.30 & 3.37 & 3.13 & 3.49 \\
\hline $\mathrm{K}_{2} \mathrm{O}$ & 0.90 & 1.01 & 1.12 & 1.21 & 1.29 \\
\hline $\mathrm{P}_{2} \mathrm{O}_{5}$ & 0.08 & 0.05 & 0.01 & 0.05 & 0.15 \\
\hline TOTAL & 97.64 & 99.78 & 99.78 & 99.91 & 98.49 \\
\hline LOI & 0.82 & 0.53 & 2.97 & 3.21 & 1.60 \\
\hline $\mathrm{Sr}$ & 429 & 425 & 267 & 268 & 232 \\
\hline $\mathrm{Rb}$ & 30 & 28 & 26 & 18 & 22 \\
\hline $\mathrm{Ba}$ & 248 & 288 & 164 & 327 & 156 \\
\hline $\mathrm{Ni}$ & 48 & 18 & 13 & 3 & 0 \\
\hline $\mathrm{Cr}$ & 135 & 31 & 25 & 17 & 6 \\
\hline V & - & - & . & - & . \\
\hline
\end{tabular}

SULU SEA Sites $769 \mathrm{C}$ and $771 \mathrm{~A}$

\begin{tabular}{lcrrr}
\hline & $769 \mathrm{C}$ & $769 \mathrm{C}$ & $769 \mathrm{C}$ & \multicolumn{1}{c}{$771 \mathrm{~A}$} \\
& $\begin{array}{c}7 \mathrm{R}-1 \\
118-120 \mathrm{~cm}\end{array}$ & $\begin{array}{c}9 \mathrm{R}-3 \\
110-113 \mathrm{~cm}\end{array}$ & $\begin{array}{c}9 \mathrm{R}-4 \\
49-50 \mathrm{~cm}\end{array}$ & $\begin{array}{c}11 \mathrm{R}-2 \\
91-94 \mathrm{~cm}\end{array}$ \\
& & & & \\
& & & & \\
$(\mathrm{wt} \%)$ & & & 52.40 & 46.90 \\
$\mathrm{SiO}_{2}$ & 55.70 & 58.40 & 0.98 & 0.68 \\
$\mathrm{TiO}_{2}$ & 0.99 & 0.97 & 19.35 & 14.66 \\
$\mathrm{Al}_{2} \mathrm{O}_{3}$ & 17.01 & 16.31 & 7.05 & 9.59 \\
$\mathrm{Fe}_{2} \mathrm{O}_{3}$ & 7.57 & 7.46 & 0.15 & 0.23 \\
$\mathrm{MnO}$ & 0.16 & 0.16 & 3.97 & 8.56 \\
$\mathrm{MgO}$ & 3.85 & 3.36 & 8.27 & 12.35 \\
$\mathrm{CaO}$ & 7.70 & 6.81 & 3.52 & 1.56 \\
$\mathrm{Na}_{2} \mathrm{O}$ & 3.23 & 3.25 & 1.78 & 0.18 \\
$\mathrm{~K}_{2} \mathrm{O}$ & 1.22 & 1.45 & 0.40 & 0.10 \\
$\mathrm{P}_{2} \mathrm{O}_{5}$ & 0.30 & 0.25 & 100.30 & 99.72 \\
$\mathrm{TOTAL}$ & 99.51 & 100.06 & 2.43 & 4.91 \\
$\mathrm{LOI}$ & 1.78 & 1.64 & & \\
$(\mathrm{ppm})$ & & & 308 & 293 \\
$\mathrm{Sr}$ & 273 & 248 & 37 & 3 \\
$\mathrm{Rb}$ & 28 & 32 & 17 & 35 \\
$\mathrm{Ba}$ & 158 & 145 & 145 & 105 \\
$\mathrm{Ni}$ & 31 & 20 & 35 & 221 \\
$\mathrm{Cr}$ & 47 & 16 & 41 & 278 \\
$\mathrm{~V}$ & 223 & 228 & 238 & \\
\hline
\end{tabular}

MINDANAO

\begin{tabular}{|c|c|c|c|c|c|c|c|c|c|c|c|c|c|c|}
\hline \multirow{4}{*}{$\begin{array}{c}\text { (a) } \\
\text { Sample }\end{array}$} & \multicolumn{4}{|c|}{-Young Volcanoes - } & \multicolumn{3}{|c|}{-Surigao- } & \multicolumn{2}{|c|}{ S. Davao- } & \multicolumn{2}{|c|}{$\begin{array}{r}-\mathrm{C} \text {. } \\
\text { Cordillera }\end{array}$} & \multicolumn{3}{|c|}{-Daguma Range } \\
\hline & 14 & 13 & 12 & 11 & 10 & 9 & 4 & 8 & 7 & & 3 & 5 & 2 & 1 \\
\hline & MNO & MNO & MNO & MNO & MNO & MNO & MNO & MNO & MNO & MNO & MNO & MNO & MNO & MNO \\
\hline & $89-16 \mathrm{~B}$ & $89-16 A$ & $89-37$ & $89-17$ & $88-46$ & $88-49$ & $88-54$ & $89-21 \mathrm{~A}$ & 89-21B & $89-12$ & $89-20$ & $89-25$ & $89-26 \mathrm{~A}$ & $89-26 B$ \\
\hline \multicolumn{15}{|l|}{ (wt \%) } \\
\hline $\mathrm{SiO}_{2}$ & 49.80 & 52.50 & 54.00 & 53.50 & 65.65 & 58.00 & 49.50 & 63.90 & 60.70 & 55.30 & 60.85 & 49.50 & 55.35 & 60.20 \\
\hline $\mathrm{TiO}_{2}$ & 1.07 & 1.01 & 0.93 & 1.42 & 0.42 & 0.53 & 0.80 & 0.30 & 0.42 & 0.87 & 0.69 & 1.14 & 1.06 & 0.82 \\
\hline $\mathrm{Al}_{2} \mathrm{O}_{3}$ & 15.22 & 18.89 & 17.38 & 16.38 & 16.20 & 18.26 & 17.50 & 16.16 & 17.16 & 18.18 & 16.72 & 17.76 & 16.67 & 16.57 \\
\hline $\mathrm{Fe}_{2} \mathrm{O}_{3}$ & 8.55 & 7.82 & 9.67 & 8.55 & 3.08 & 6.60 & 9.69 & 3.65 & 4.66 & 7.46 & 5.29 & 10.19 & 9.90 & 6.18 \\
\hline $\mathrm{MnO}^{3}$ & 0.14 & 0.14 & 0.19 & 0.13 & 0.06 & 0.16 & 0.18 & 0.08 & 0.10 & 0.16 & 0.07 & 0.19 & 0.20 & 0.17 \\
\hline $\mathrm{MgO}$ & 9.60 & 3.01 & 4.06 & 5.87 & 2.05 & 2.45 & 4.10 & 0.98 & 1.99 & 3.85 & 2.11 & 3.82 & 3.39 & 2.31 \\
\hline $\mathrm{CaO}$ & 8.55 & 7.46 & 8.23 & 7.50 & 3.46 & 7.00 & 10.18 & 2.62 & 3.87 & 8.24 & 4.69 & 6.98 & 7.50 & 7.24 \\
\hline $\mathrm{Na}_{2} \mathrm{O}$ & 3.09 & 3.65 & 3.11 & 3.60 & 5.30 & 4.08 & 2.61 & 4.86 & 4.86 & 3.04 & 3.66 & 4.37 & 4.11 & 3.10 \\
\hline $\mathrm{K}_{2} \mathrm{O}$ & 1.30 & 3.06 & 1.78 & 0.98 & 2.77 & 1.31 & 1.32 & 3.52 & 2.38 & 0.65 & 2.42 & 0.54 & 0.67 & 0.16 \\
\hline $\mathrm{P}_{2} \mathrm{O}_{5}$ & 0.30 & 0.75 & 0.40 & 0.30 & 0.15 & 0.15 & 0.25 & 0.15 & 0.25 & 0.30 & 0.30 & 0.08 & 0.05 & 0.05 \\
\hline TOTAL & 98.95 & 98.97 & 99.77 & 99.22 & 100.06 & 99.80 & 99.83 & 99.20 & 99.43 & 99.65 & 99.22 & 99.13 & 99.49 & 99.01 \\
\hline $\begin{array}{l}\text { LOI } \\
\text { (ppm) }\end{array}$ & 1.33 & 0.68 & 0.02 & 0.99 & 0.92 & 1.26 & 3.66 & 2.98 & 3.04 & 1.60 & 2.42 & 4.66 & 0.59 & 2.21 \\
\hline $\mathrm{Sr}$ & 578 & 643 & 640 & 540 & 946 & 506 & 583 & 303 & 555 & 320 & 411 & 303 & 198 & 251 \\
\hline $\mathrm{Rb}$ & 20 & 72 & 34 & 16 & 42 & 22 & 11 & 72 & 42 & 20 & 49 & 9 & 10 & 2 \\
\hline $\mathrm{Ba}$ & 215 & 635 & 392 & 184 & 624 & 367 & 109 & 725 & 527 & 132 & 580 & 45 & 122 & 66 \\
\hline $\mathrm{Ni}$ & 236 & 17 & 13 & 83 & 31 & 4 & 15 & - & 14 & 23 & - & 5 & 4 & - \\
\hline $\mathrm{Cr}$ & 426 & 35 & 16 & 142 & 48 & 4 & 41 & - & 28 & 39 & - & 3 & 3 & $=$ \\
\hline V & 140 & 230 & 260 & 175 & 50 & 140 & 270 & 60 & 85 & 180 & 120 & 260 & 260 & 140 \\
\hline
\end{tabular}


Table 3. Summary of data on onshore magmatism including: rock-types deduced from Table 2 and Figures $3 \mathrm{~A}$ and $3 \mathrm{~B}$, isotopic ages from Table 1, field locations and site sampling numbers from Figure 2 .

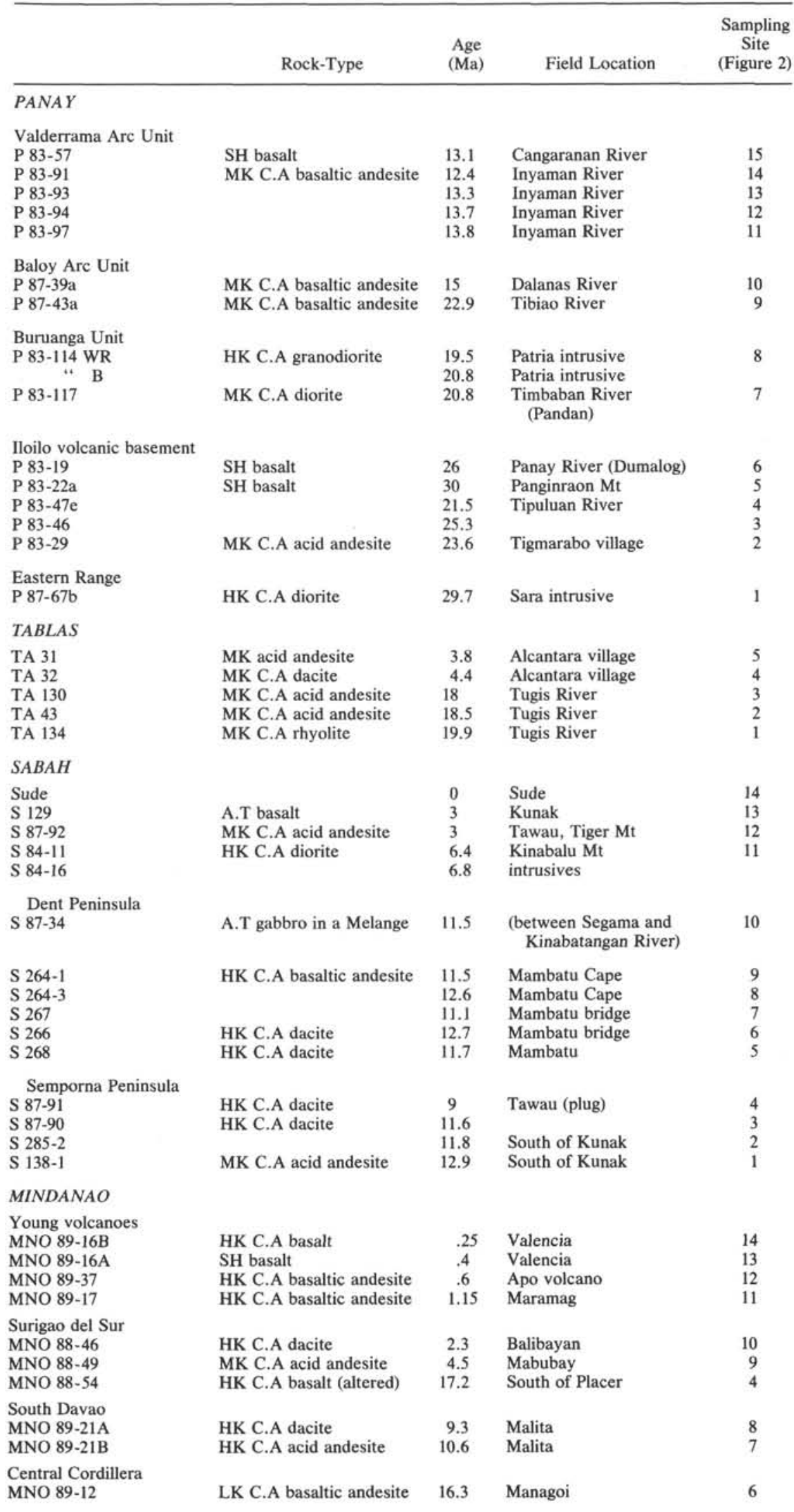


Table 3 (continued).

\begin{tabular}{|c|c|c|c|c|}
\hline & Rock-Type & $\begin{array}{l}\text { Age } \\
\text { (Ma) }\end{array}$ & Field Location & $\begin{array}{c}\text { Sampling } \\
\text { Site } \\
\text { (Figure 2) }\end{array}$ \\
\hline MNO $89-20$ & MK C.A acid andesite & 19.9 & Tangkulang Mt & 3 \\
\hline $\begin{array}{l}\text { Daguma Range } \\
\text { MNO 89-25 } \\
\text { MNO 89-26A } \\
\text { MNO 89-26B }\end{array}$ & $\begin{array}{l}\text { LK C.A basalt } \\
\text { A.T diorite } \\
\text { LK C.A diorite }\end{array}$ & $\begin{array}{l}16.7 \\
29.9 \\
31.9\end{array}$ & $\begin{array}{l}\text { Salonic } \\
\text { Talub } \\
\text { Talub }\end{array}$ & $\begin{array}{l}5 \\
2 \\
1\end{array}$ \\
\hline \multicolumn{5}{|l|}{ MASBATE } \\
\hline $\begin{array}{l}\text { MAS } 88-69 \\
\text { MAS } 88-79 \text { A }\end{array}$ & $\begin{array}{l}\text { HK C.A acid andesite } \\
\text { HK C.A basaltic andesite }\end{array}$ & $\begin{array}{r}9.7 \\
10.6\end{array}$ & $\begin{array}{l}\text { Along the } \\
\text { Sibuyan Sea fault }\end{array}$ & $\begin{array}{l}2 \\
1\end{array}$ \\
\hline \multicolumn{5}{|c|}{ NORTH SULAWESI } \\
\hline $\begin{array}{l}\text { SN 89-01 } \\
\text { SN 89-32 } \\
\text { SN } 89-47 \\
\text { SN } 89-33 \\
\text { SN } 89-46 \\
\text { SN } 89-12\end{array}$ & $\begin{array}{l}\text { MK C.A andesite } \\
\text { MK C.A dacite } \\
\text { LK C.A andesite } \\
\text { HK C.A granodiorite } \\
\text { HK C.A granite } \\
\text { HK C.A granodiorite }\end{array}$ & $\begin{array}{r}4.1 \\
4.4 \\
6.9 \\
8.8 \\
18.5 \\
22.2\end{array}$ & $\begin{array}{l}\text { North of Bilungala } \\
\text { East of Bilungala } \\
\text { West of Gorontalo } \\
\text { Between Mariza } \\
\text { and Gorontalo } \\
\text { Southeast of Soronga }\end{array}$ & $\begin{array}{l}6 \\
5 \\
4 \\
3 \\
2 \\
1\end{array}$ \\
\hline
\end{tabular}

Abbreviations for rock-types: A.T., arc tholeiitic series; C.A., calc-alkaline series with LK: low-K calc-alkaline rock; MK, medium-K calc-alkaline; HK, high-K calc- alkaline; $\mathrm{SH}$, shoshonitic series.

Serawagan pillow basalts (from southwestern Panay) described by Santa Cruz et al. (1989). The shoshonitic basalt sample from Valderrama has a higher $\mathrm{TiO}_{2}$ content $(1.27 \%)$ and a lower Ba content (333 ppm).

\section{Conclusions}

The isotopic ages obtained on orogenic lavas and plutonic bodies on Panay Island allow one to distinguish three main diachronous episodes of arc activity:

1. The oldest ages belong to the Iloilo volcanic and plutonic basement with a magmatic activity ranging in age from $30 \mathrm{Ma}$ (or even before) to $21.5 \mathrm{Ma}$.

2. The second episode is concentrated in the Buruanga and the Mt. Baloy units of northern Panay. It stops around $15 \mathrm{Ma}$. The oldest ages for the Mt. Baloy unit slightly overlap the youngest ages for the Iloilo basement.

3 . These volcanic units are spatially and tectonically separated by the Valderrama unit that appears as a younger (13.9-12.4 Ma) and a distinct volcanic episode.

\section{TABLAS}

Recent field work on Tablas Island (Marchadier, 1988; Marchadier and Rangin, 1989) has shown that a large and thick volcaniclastic series overlies a metamorphic basement. It forms a north-south-trending dome across the whole island and it consists of a lower volcanic sequence conformably overlain by a volcaniclastic sequence and pillow basalts, and minor volcaniclastic sediments containing Zones NN6-NN9 calcareous nannoplankton. Two acid andesites and a dacite recovered at the base of the lower level of the central part of the island (Tugis River) yield ages of 19.9-18 Ma. A second volcaniclastic sequence overlies disconformably the metamorphic basement in the southern part of the island, south to Alcantara Village. An andesite and a dacite from this sequence have yielded more recent ages of 4.4 and $3.8 \mathrm{Ma}$. Isotopic dates clearly show that two arc episodes have occurred in Tablas.

One can note the overall similarity in geochemistry of the calc-alkaline acid andesites of both episodes (Table 2 and Fig. 3A).
The oldest volcanic episodes that ceased between 18 and 14 Ma (Zone NN6) can be compared with the Mt. Baloy volcanic sequence in Panay.

The early Miocene volcanic belt extending from Tablas to northern Panay Island can be traced offshore in the Sulu Sea along the Cagayan Ridge (Fig. 2).

\section{ISOTOPIC AGES OF VOLCANIC ROCKS DRILLED ALONG THE CAGAYAN RIDGE}

The east-northeast submerged Cagayan Ridge within the Sulu Sea separates the outer basin from the inner one. This ridge was the locus of volcanic activity that has emitted basalts and porphyritic andesites. Their chemical compositions indicate that this ridge originated as a volcanic arc. Isotopic dates were determined on dredged volcanic rocks (Kudrass et al., 1986; Kudrass et al., 1990) and have yielded the following results:

1. At the southern end of the ridge, near Meander Reef, a basaltic andesite is dated $14.7 \pm 0.6 \mathrm{M} \alpha$

2 . Volcanic rocks at its northern end are dated 20-14 Ma (Site SO 49-55) or much older (SO 49-59); whole-rock ages of a porphyritic andesite and a dacite range from 50.5 to $22.0 \mathrm{Ma}$ and separated minerals yield ages as old as 158,60 , and $36 \mathrm{Ma}$ for amphiboles and 25.8-23.9 Ma for plagioclase. If results for amphibole are interpreted as proving their xenolithic character (metamorphic basement mechanically mobilized by volcanism) (Kudrass et al., 1990), one may remark that Site SO 49-59 is not far from the axis of a large canyon flowing from southern Panay (Rangin and Silver, this volume). Consequently, these dredged lavas can belong to southern Panay island where older volcanic rocks outcrop.

These data have been completed with ages determined on five drilled lavas during Leg 124, at ODP Sites 769 and 771, and listed in Table 1. Four lavas have been analyzed for major and trace elements (Table 2). Results are plotted in the $\mathrm{K}_{2} \mathrm{O}$ vs. $\mathrm{SiO}_{2}$ diagram (Fig. 3A) and two rocks have been selected for their normalized incompatible elements patterns (Figs. 4A and 4B).

Site 769 was drilled in the southeastern flank of the Cagayan Ridge. Volcaniclastic material was encountered at 285 mbsf. Upper sediments that rest on it are early Miocene to early middle Miocene in age. Volcaniclastic material consists of massive and 


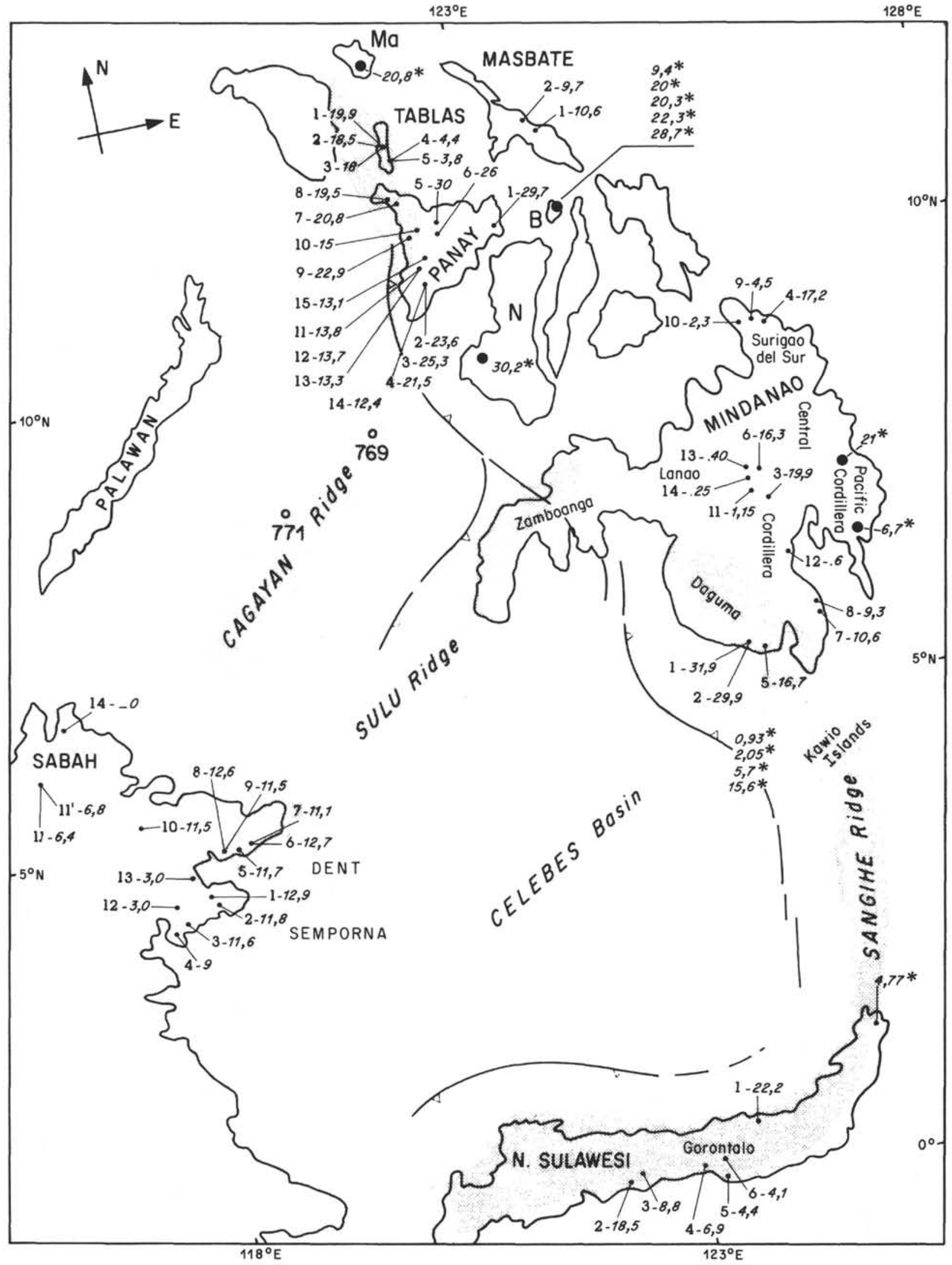

Figure 2. Map of the study area around the Sulu and Celebes basins with the location of the sampling (first number) and the K-Ar isotopic ages (second number) taken from Table 1. Asterisks indicate isotopic dates previously published: by Wolfe (1981) on Bantayan (B) and Mindanao; by Walther et al. (1981) on Marinduque (Ma) and Negros (N); by Morrice et al. (1983) on the Kawio Islands and Sulawesi. 
A

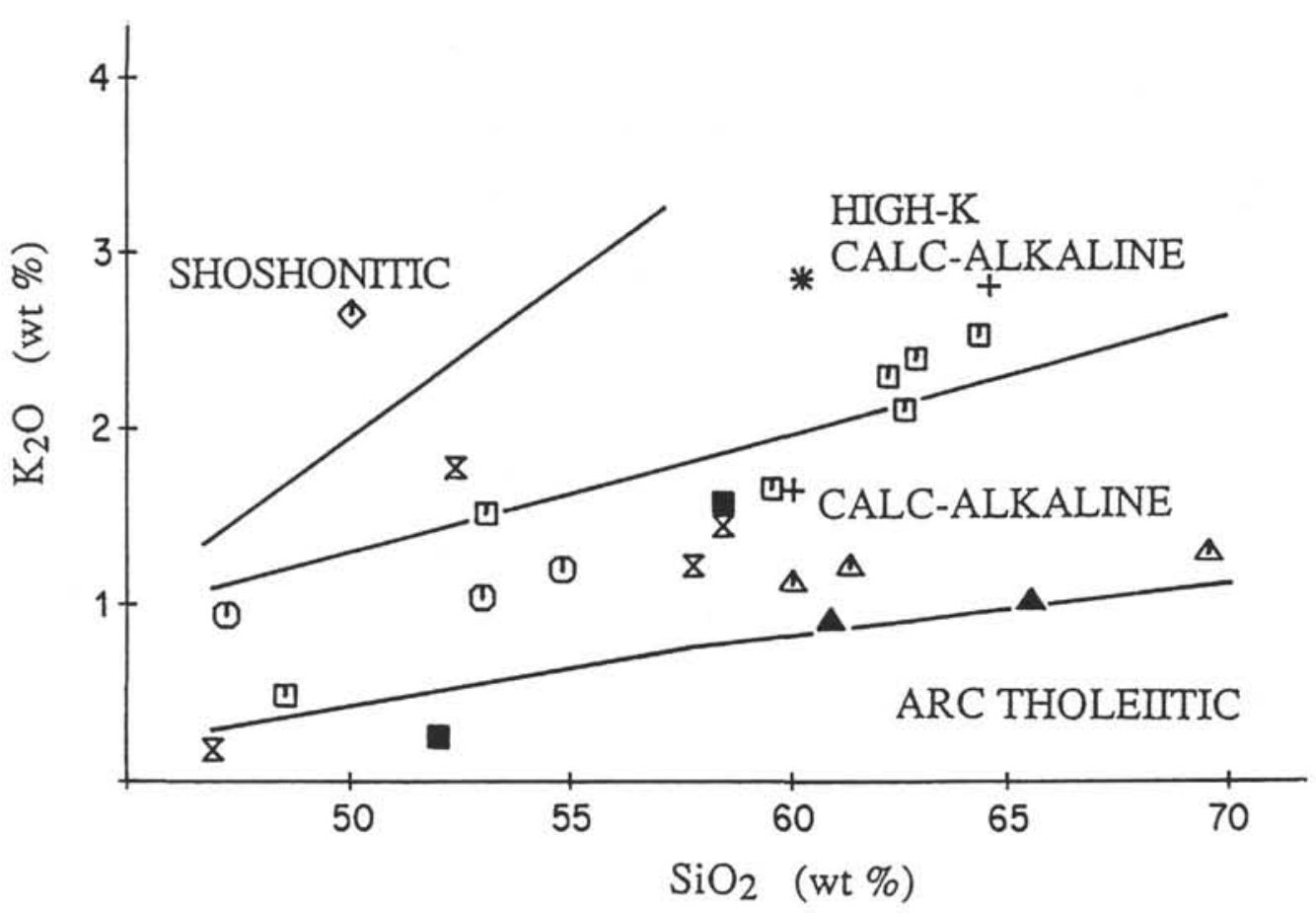

TABLAS

$=4 \mathrm{Ma}$

$=20-18 \mathrm{Ma}$

$\Delta$

WESTERN PANAY

Valderrama

Baloy

Buruanga

(1)

$+$

SABAH

$<3 \mathrm{Ma}$

Kinabalu

$=13-9 \mathrm{Ma}$

籴

CAGAYAN RIDGE $\square$

B

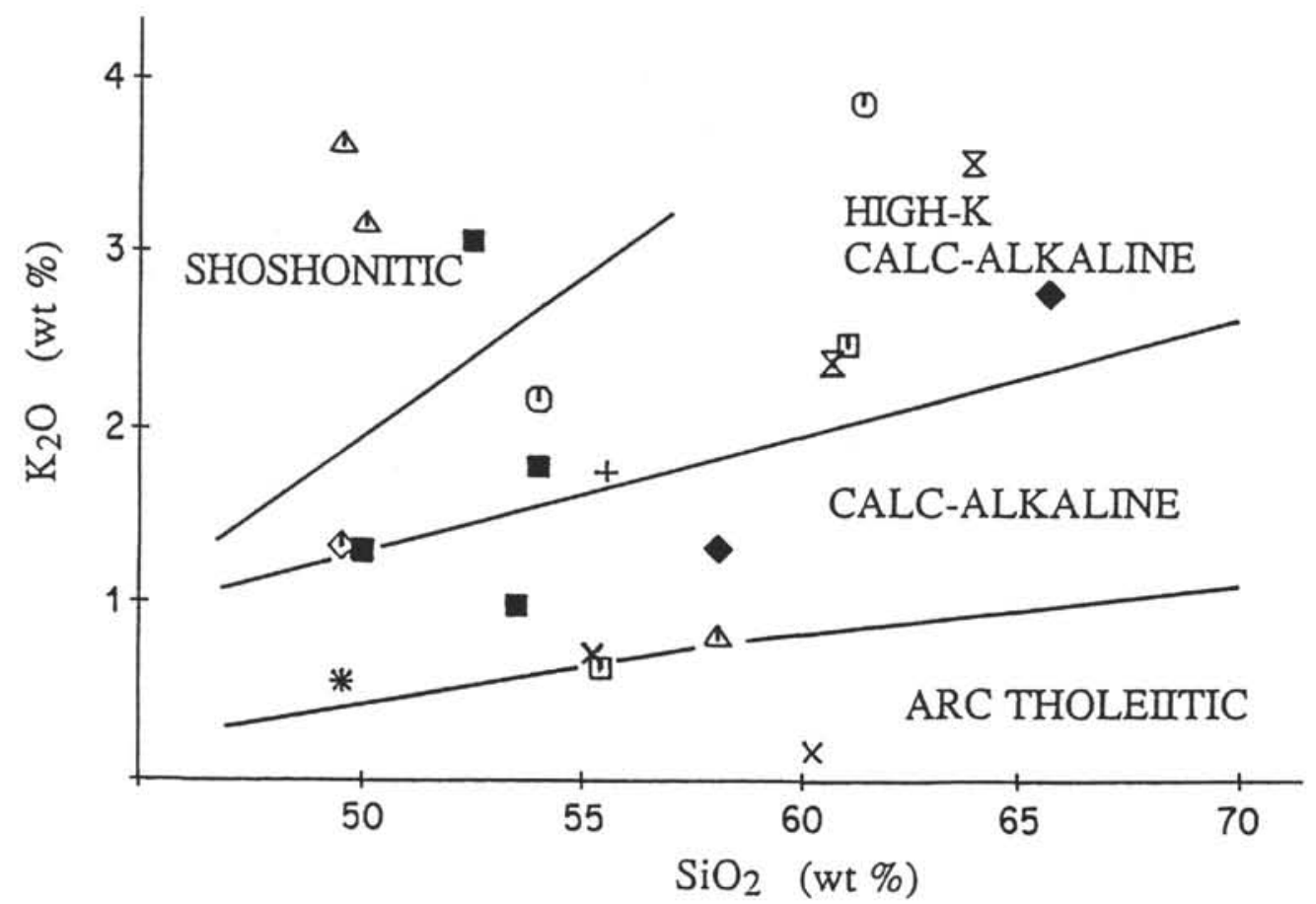

EASTERN PANAY

Eastern Range +

Iloilo

$\Delta$

MASBATE

(1)

MINDANAO

Central Cordillera

$<1,2 \mathrm{Ma}$

$=20-16 \mathrm{Ma}$

Surigao

$=2,3-4,5 \mathrm{Ma}$

$=17,2 \mathrm{Ma}$

South Davao

ए

Daguma

Salonic

Talub

$$
\begin{aligned}
& + \\
& \Delta \\
& 0
\end{aligned}
$$

Figure 3. Graph of $\mathrm{K}_{2} \mathrm{O}$ vs. $\mathrm{SiO}_{2}$ for 43 dated samples. Superimposed fields are from Peccerillo and Taylor (1976) modified after Maury (1984). A: samples from western Panay, Tablas, Sabah, and Cagayan Ridge. B: samples from central and eastern Panay, Mindanao, and Masbate. Full symbols represent samples younger than $8 \mathrm{Ma}$. 

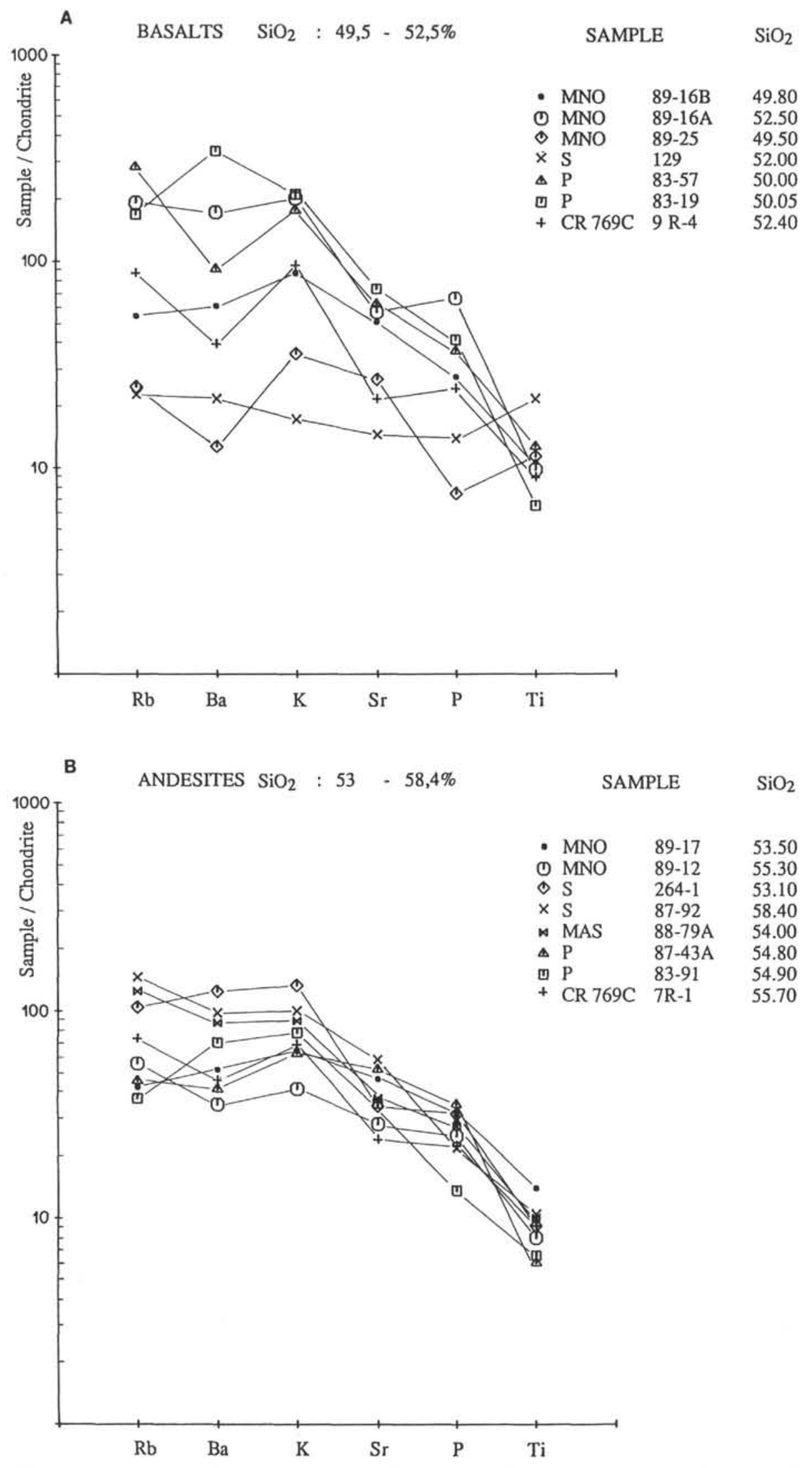

Figure 4. Comparison of normalized incompatible elements ( $\mathrm{Rb}, \mathrm{Ba}, \mathrm{K}, \mathrm{Sr}, \mathrm{P}$ and $\mathrm{Ti}$ ) patterns for selected basalts (A) and andesites (B). Abundances are normalized using chondritic abundances from Wakita et al. (1971) and from Sun et al. (1979): Rb, 0.35; Ba, 3.51; K, 120; Sr, 11; P, 46; and $\mathrm{Ti}, 600 \mathrm{ppm}$. 


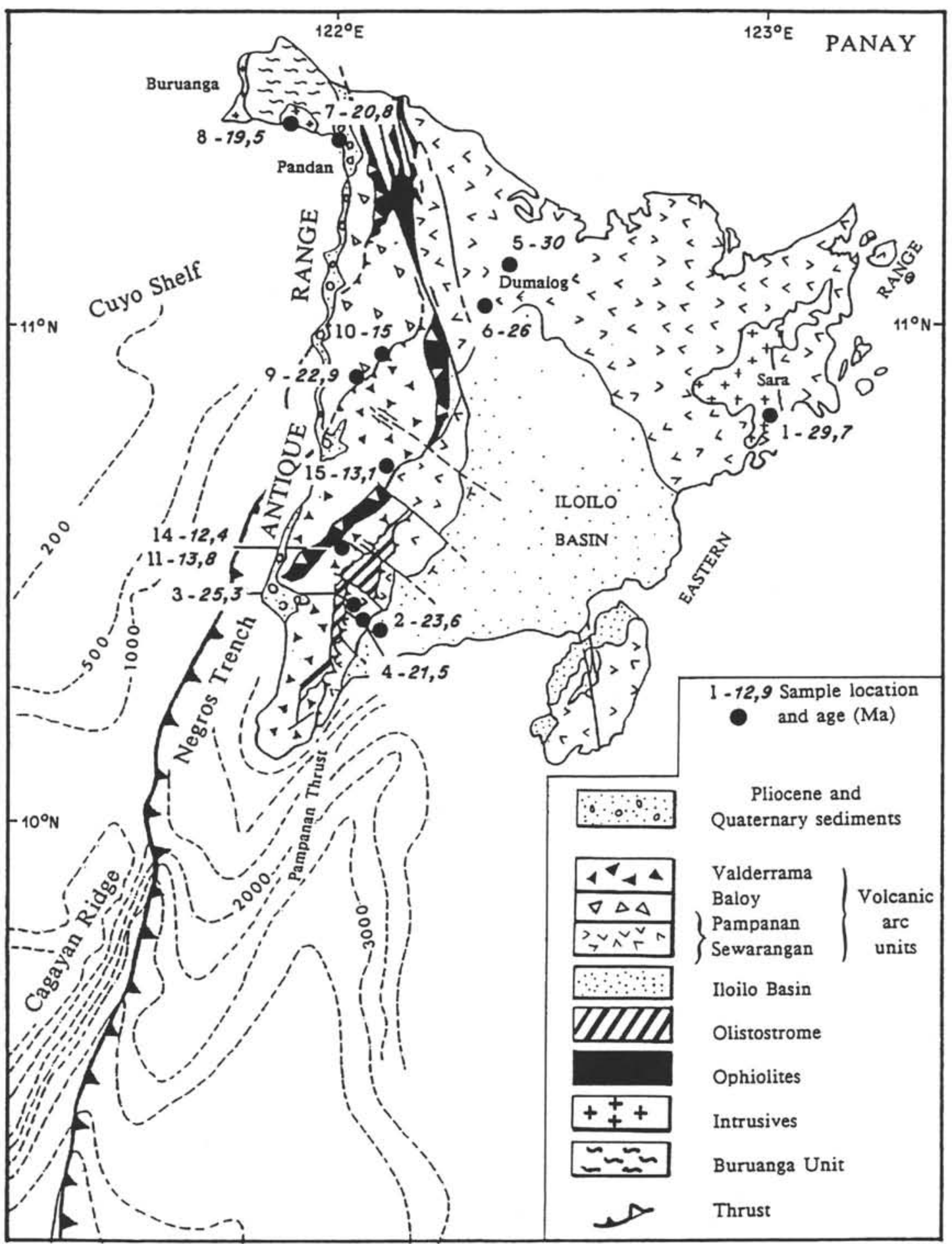

Figure 5. Schematic geologic map of Panay (from Rangin et al., in press) with indications of sampling localities and $\mathrm{K}$-Ar isotopic dates taken from Table 1. It shows both the structural and age relationships between the arc units in western Panay and the Iloilo basement in central Panay.

unstratified lapillistone and coarse tuff. Volcanic clasts are mainly porphyritic andesites with plagioclase, clinopyroxene, and olivine or orthopyroxene. Rock aliquotes were sampled between 319 and 328.6 mbsf (Core 124-769-7R-1), and between 338.2 and 347.9 mbsf (Cores 124-769-9R-3 and -9R-4).

Among the three analyzed lavas, the basaltic andesites (Sample 124-769-9R-4, 49-50 cm) is more altered than the andesites (Sample 124-769-7R-1, 118-120 cm, and -9R-3,
$110-113 \mathrm{~cm}$ ). It has a significantly higher LOI (loss on ignition) $(2.43 \%)$ and exhibits a brown oxidized glass and a larger developed secondary mineralogy.

These facts increase confidence in the mean isotopic dates of 20.6 and 19.8 Ma for the andesites, and of $14.7 \mathrm{Ma}$ for the basaltic andesites as a minimum age.

Site 771 was drilled in the Cagayan Ridge, on the large plateau along its southern flank, $50 \mathrm{~km}$ southwest of Site 769 . 


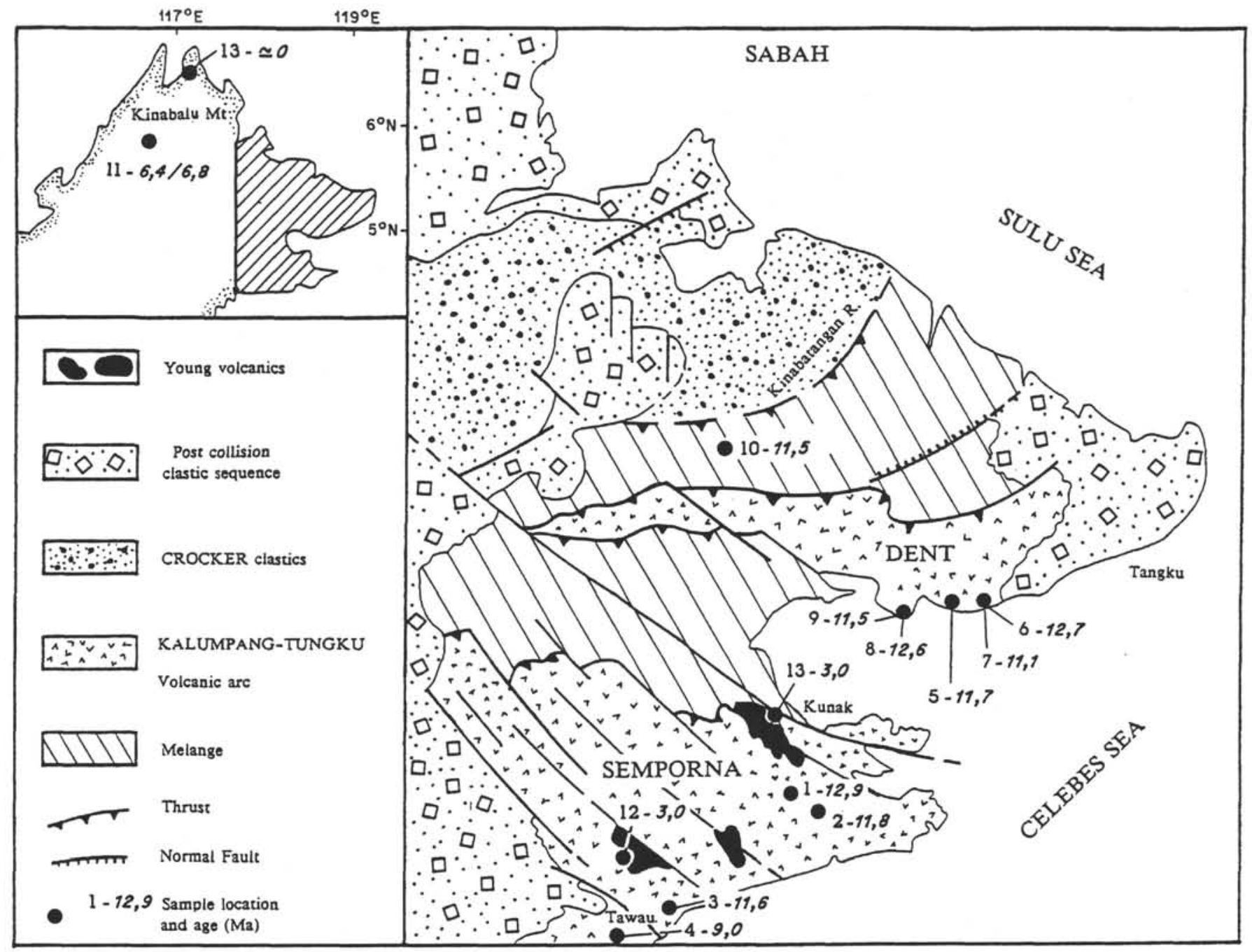

Figure 6. Schematic geologic map of eastern Sabah and location of Kinabalu intrusives (cartridge). K-Ar isotopic dates are taken from Table 1.

The hole has penetrated, between 233.9 and 303 mbsf, a volcanic unit consisting of massive lapillistone, tuff, and probable basaltic flows. The upper unit is middle Miocene to late Pliocene in age and is made of sediments (clays and marl) with interbedded altered ash layers.

Sample 124-771-11R-2, 91-94 cm, from a basaltic flow seated between 232 and 241.7 mbsf, is a low $\mathrm{K}_{2} \mathrm{O}(0.22 \%)$ and low $\mathrm{TiO}_{2} \mathrm{LOI}\left(1050^{\circ} \mathrm{C}\right)(0.68 \%)$ vesicular basalt of orogenic parentage (arc tholeiitic basalt) but unfortunately has high LOI $(4.91 \%)$. Vesicles are partly filled with silica.

Sample 124-771-18R-7, 142-144 cm (294.4-304.1 mbsf), is from an andesitic block among andesitic angular clasts in a massive lapillistone. Its LOI was not measured but its undervacuum outgassing spectrum is two times lower than that of the arc tholeiitic basalt from section 11R-2, suggests its lower LOI.

These two lavas yield discordant isotopic ages (Table 1); respectively $19.9 \mathrm{Ma}$ for the basalt and $14 \mathrm{Ma}$ for the andesite. Taking account of the better preservation of the andesite, we are more certain of a 14-Ma age for the activity at this site where the oldest mudstone layer within the pyroclastics was dated upper Zone NN3 and the youngest pyroclastics, Zone NN5 (Rangin and Silver, this volume). This last age is in accordance with the youngest isotopic age determined at this site.
In conclusion, the set of isotopic dates compared with the stratigraphic ages allows us to propose an activity for the Cagayan Ridge that spans 20-14 Ma. Two stages can be tentatively distinguished.

\section{SABAH}

\section{Geological Overview}

Sabah (northern Borneo)(Fig. 6) is bordered to the east by the Sulu Sea and to the north by an active subduction or collision zone, the Palawan-north Borneo Trench (Hamilton, 1979). Its Tertiary Belt is considered to be part of an accretionary prism related to the Palawan Trench. This orogenic belt consists of an outer belt (Crocker Range) and an inner belt made of terranes of central and southern Sabah that overthrusts the outer belt.

The Crocker Range is dominantly sedimentary; calcareous nannofossils from limestones give late middle Eocene to Oligocene ages; i.e., Zones NP17-NP20 in Kudat Peninsula, Zone NP24 in Banggi Island and in central eastern Sabah, north of the Kinabatangan River. Sediments with Zones NN5 and NN6 nannoplankton of lower middle Miocene were found in Kudat Peninsula and in the northern islands. 
The inner belt consists of large deformed volcanic and sedimentary sequences that outcrop in the Semporna and Dent Peninsulas. They are imbricated with ophiolites and chaotic formations. The different volcanic facies are irregularly distributed in the Semporna Peninsula; tuffs and pyroclastites are mainly present in the northern slices, and massive flows and breccias are widely distributed around Tawau (Rangin et al., 1990b). The sequence is folded with a general northeast trend that curves to the east-southeast at the Dent Peninsula. Nannofossil ages from the sedimentary levels range from late Oligocene, Zone NP24, to early middle Miocene, Zone NN5. A similar folded volcanic assemblage is present in the Dent Peninsula.

\section{Isotopic Ages}

Massive lava flows were selected for isotopic dating along the southern coast of Dent Peninsula and in Semporna Peninsula south of Kunak and close to Tawau (Fig. 6 and Table 3). A gabbro from the inner belt melange was also selected. Among ten dates, nine of them fall within the interval 12.911.1 Ma. All these rocks are within folded sequences. Only the youngest sample $(9 \mathrm{Ma})$ is distinct from these sequences and was taken from a plug near Tawau. To these results may be added younger ages obtained on Mount Kinabalu intrusives (6.8 and $6.4 \mathrm{Ma}$ ), the highest peak in Borneo, on volcanism injected along N 130 E-trending faults in the Kunak area (Ma) and on volcanics that outcrop at the northern end of Sabah (Sude with a near 0-Ma age). Ages of $3 \mathrm{Ma}$ (with a large error) remain suspect because of the high amount of ${ }^{36} \mathrm{Ar}$ isotope in these fresh lavas without alteration. Such a concentration may reflect strong fluid circulations within the volcanic pile, the ${ }^{40} \mathrm{Ar} /{ }^{36} \mathrm{Ar}$ isotopic ratio of it being possibly greater than the atmospheric one. In this case, the applied atmospheric correction is incorrect and leads to ages older than the geological age. From these results, we conclude that these injected volcanics along $\mathrm{N} 130 \mathrm{E}$ faults are probably very young and, in consequence, the $\mathrm{N} 130 \mathrm{E}$ faults are still active.

\section{Geochemistry}

Andesites and dacites dated 12.9-9 Ma plot into the $\mathrm{K}-2 \mathrm{O}-\mathrm{SiO}_{2}$ diagram (Fig. 3A) along the limit of the calcalkaline and the high-K calc-alkaline field and within the high-K field. The normalized incompatible elements pattern (Fig. 4B) for one andesite (S 264-1) shows its high Rb, Ba, and $\mathrm{K}$ concentrations ( $>100$ times the chondritic values).

The gabbro from the melange has a typical low-K arc tholeiitic basalt composition using the $\mathrm{FeO}^{*} / \mathrm{MgO}$ ratio criterion (Gill, 1981). Arc tholeiitic and calc-alkaline suites are associated in space and time.

Younger rocks display large variations of composition. Kinabalu intrusive plots in the high-K calc-alkaline field. A previous detailed study (Vogt and Flower, 1989) pointed out that the high-K character was developed during high-pressure sialic contamination of a low-K type melt that forms the small central core of the batholit.

Volcanoes younger than $3 \mathrm{Ma}$ are of arc tholeiitic and of calc-alkaline parentages.

\section{MINDANAO}

Until now, dates on samples from Mindanao were few and poorly located (Wolfe, 1981). Except for the surprising ages ranging from Ordovician to Early Cretaceous of the Bislig quartz diorite (Surigao del Sur), all the other ages are younger. One is $60 \mathrm{Ma}$ (diorite dike at $7^{\circ} 30^{\prime} \mathrm{N}, 126^{\circ} 13^{\prime} \mathrm{E}$ ) and the others are 21,11 , and $6.7 \mathrm{Ma}$, so in the range of the new ages reported here (Table 1). The 11-Ma date remains unlocated, the 21-Ma date was tentatively attributed to volcanics located east of Compostela $\left(7^{\circ} 42^{\prime} \mathrm{N}, 126^{\circ} 10^{\prime} \mathrm{E}\right)$ in the Pacific cordillera, and the $6.7-\mathrm{Ma}$ age is that of a probable andesite at the southeastern tip of the island.

Fourteen new isotopic dates (Tables 1 and 3) range between 32 and $0.2 \mathrm{Ma}$. These results are preliminary because field work is in progress, but they fall into five main groups: $32-30 \mathrm{Ma}$; 20-16 Ma; $11-9 \mathrm{Ma}$; 4.5-2.3 Ma; and less than 1.2 Ma. Oldest ages are restricted to the Daguma Range, where, near Talub, dioritic to granodioritic blocks of arc tholeiitic parentage (MNO 89-26A) and of low-K calcalkaline parentage (MNO 89-26B) (Table 2, Fig. 3B) and associated with altered "andesites", yield ages of 31.9 and $30 \mathrm{Ma}$. They geochemically resemble the Sipalay Mine tonalite in western Negros dated at $30.2 \mathrm{Ma}$ (Walther et al., 1981).

Four samples, one from the Daguma Range, two from the central cordillera, and the last from Surigao del Sur, yield ages that fall between 20 and $16 \mathrm{Ma}$. Lavas dated 16.7 and $16.2 \mathrm{Ma}$ are from a low-K calc-alkaline series. Oldest lavas $(20 \mathrm{Ma})$ are from a middle- $K$ calc-alkaline series. Two different stages of arc activity can be ascertained here.

An andesite and a dacite with ages of 10.6 and $9.3 \mathrm{Ma}$ outcrop in South Davao. These lavas belong to a high-K calc-alkaline series as the andesites dated 10.6 and $9.7 \mathrm{Ma}$, which outcrop along the Sibuyan Sea Fault on the northeastern coast of Masbate (Table 1).

As in Tablas Island, activity renewed around $4.5 \mathrm{Ma}$ in Surigao del Sur and perhaps earlier $(6.7 \mathrm{Ma})$ at the southeastern tip of Mindanao, if we include the date reported by Wolfe (1981). This arc produced medium-K andesites to high-K calc-alkaline dacites (Fig. 3B).

A last volcanic event, active since $1.15 \mathrm{Ma}$, is responsible for all the volcanoes (among those, the still-active Apo Volcano) that outcrop at the western edge of the central cordillera and eastward of Lanao. One can observe from Figures $3 \mathrm{~B}$ and $4 \mathrm{~A}$ the diversity of chemical compositions of basalts and basaltic andesites (calc-alkaline, high- $\mathrm{K}$ calc-alkaline, and shoshonitic) that erupted during this recent stage.

\section{NORTH SULAWESI}

The first preliminary isotopic ages (Tables 2 and 3) together with geochemical compositions (not listed here) of plutonic bodies and lavas sampled (Fig. 2) in September 1989 around Gorontalo lead to the following interpretation: the oldest plutonic bodies are 22 to $18.5 \mathrm{Ma}$ (a period similar to that of Cagayan Ridge activity and, in Panay, of the Baloy arc sequence). This arc basement is intruded by plutonic bodies at $8.8 \mathrm{Ma}$ and covered by arc volcanics, the ages of which range from 6.9 to $4.1 \mathrm{Ma}$. At least two different arcs can be specified between 22 and $4 \mathrm{Ma}$ in North Sulawesi.

\section{CONCLUSIONS: AGE CORRELATIONS AND GEODYNAMIC IMPLICATIONS}

A synthesis of all isotopic dates from this work together with previous published ages is reported in Figure 7 and is compared with volcanic episodes in evidence offshore during Leg 124 drilling. The drilled volcanics of Cagayan Ridge, air-fall ash, and pyroclastic flows in the Sulu and Celebes Seas are considered to be a good record for the volcanic arc activity around these basins (Rangin, Silver, von Breymann, et al., 1990; Pubellier et al., this volume).

The period 32-0 Ma was only considered because the problem of preservation of older tephras in the basins remains difficult. $32 \mathrm{Ma}$ is also the oldest ${ }^{40} \mathrm{~K}-{ }^{40} \mathrm{Ar}$ age obtained onshore on this volcanic arc sequences. Figure 7 reveals that ages are randomly distributed between 32 and 0 Ma. However, six distinct stages can be tentatively differentiated. 


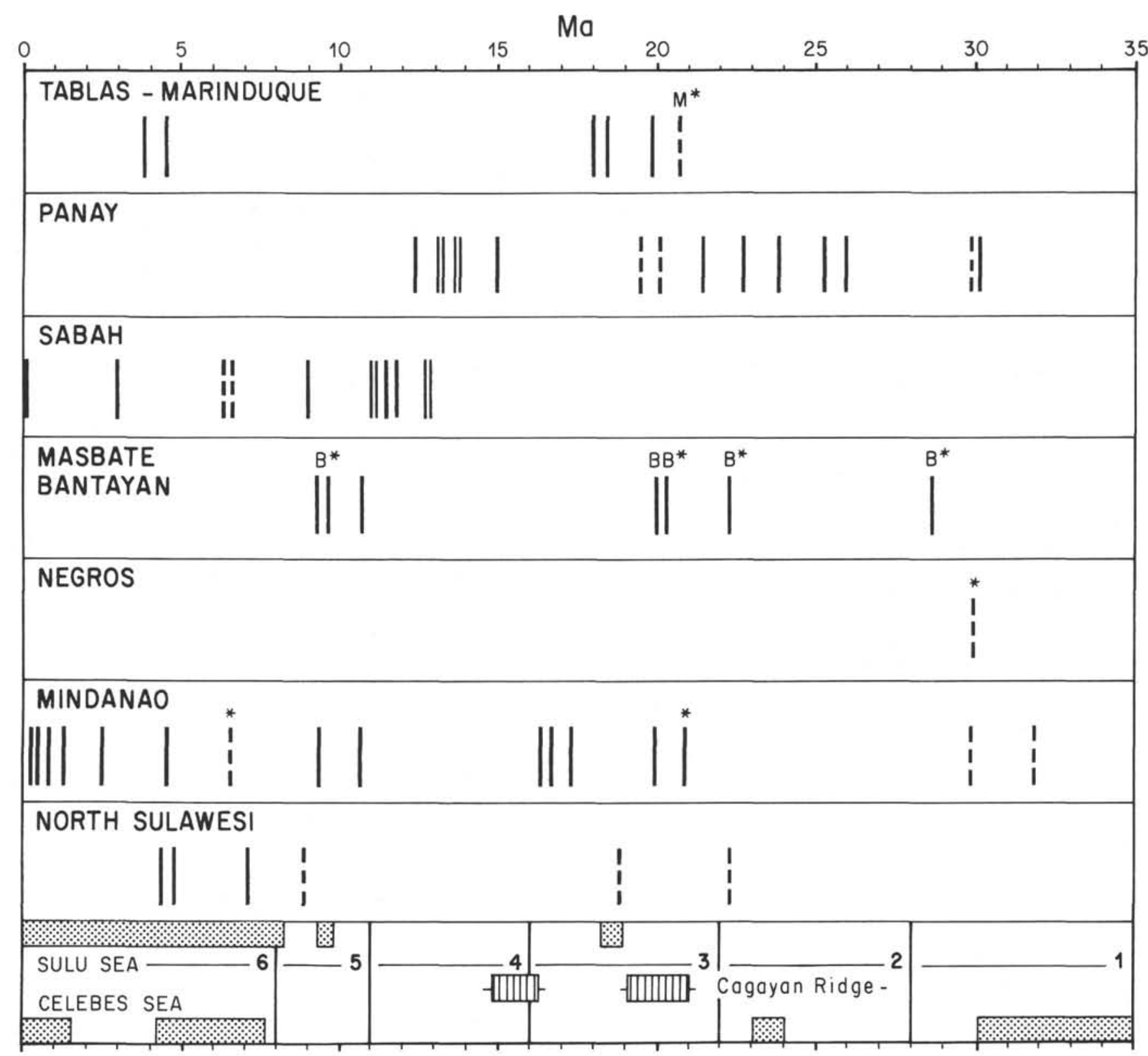

-1 a b एW] c

Figure 7. Chronological diagram of the onshore magmatic activities around the Sulu and Celebes basins and offshore volcanic activity (Cagayan Ridge). Data are taken from Table 1, and data with $\left(^{*}\right)$ are from Walther et al. (1981) for Marinduque (M) and for Negros; from Wolfe (1981) for drilled igneous rocks in the Kabak oil well in Bantayan Island (B) (location in Fig. 2) and for Mindanao volcanics. Asterisk at the top of vertical bar marks the date taken from literature. Solid vertical bar refers to the age of volcanic rocks. Dotted vertical bar refers to the age of plutonic rocks. The lower array in the diagram gives three sets of data. Graphic symbol a: Tentatively distinct stages of activity. Graphic symbol b: Timing of air-fall ash and pyroclastic events in the Sulu and Celebes basins according to Pubellier et al. (this volume). Graphic symbol c: Ages of arc activity along the Cagayan Ridge (data taken from Table 1). 
Stage 1 (older than $28 \mathrm{Ma}$ ) is only present in the Philippine Mobile Belt (Mindanao, Negros, and the Iloilo basement in Panay). No continental basement is known for these volcanic sequences, suggesting that they belong to the intraoceanic Philippine arc.

Stages $2(28-22 \mathrm{Ma})$ and $3(22-16 \mathrm{Ma})$ are both present in the Philippine arc (Masbate, Mindanao), but also along the Cagayan volcanic arc (Panay, Cagayan Ridge, Tablas) and the north arm of Sulawesi.

Stage 3 was probably one of the most volcanically active period of Southeast Asia. Collisions of the Cagayan arc with the Palawan block to the north, and of the north Sulawesi arc with the Sula block to the south, are both marked by cessation of volcanic activity around 18-17 Ma. A second phase of volcanic activity (16-14 Ma) along the Cagayan Ridge is coeval with this collision and is marked by emplacement of the large pyroclastic aprons drilled at Sites 769 and 771 .

Stage 4, dated 16-11 Ma, was marked by medium-K calc-alkaline volcanism in Panay and Sabah and can be easily explained by incipient subduction of the Sulu Sea along the Negros and Sulu Trenches after complete collision of the Philippine arc with the Cagayan arc in the central part of the Philippine Archipelago (Tablas, Marinduque).

The 11- to 8-Ma stage (denoted 5 in Fig. 7) occurred along major strike-slip faults such as the Philippine Fault in Mindanao (north Davao) and Masbate or Tawau Fault zone in Sabah. The high-K character of this calc-alkaline magmatism suggests that it could be the product of volcanism sourced into metasomatized mantle after cessation of subduction.

Activities younger than $8 \mathrm{Ma}$ are developed within the complex tectonic framework where the three major plates of this area are strongly imbricated. Volcanism in Tablas can be easily linked with the termination of the Luzon arc; in the central part of north Sulawesi, young volcanic activity can be related to the Holocene southward subduction of the Celebes Sea. Similarly, young volcanism in Mindanao can be the result of incipient subduction along the Philippine and Cotobato Trenches. Youngest ages in Sabah are either related to fault activity (Tawau) or cooling and unroofing of major plutons (Kinabalu Mt.).

This first tentative correlation of isotopic ages for volcanic rocks collected around the Sulu and Celebes basins, with markers of this activity in the basins, allows one to understand the complex magmatic- and geodynamic-related evolutions of this area. Although preliminary, this approach needs to be reinforced by much more onshore data.

\section{ACKNOWLEDGMENTS}

We thank J. C. Philippet and J. Cotten for their respective technical assistance in mass spectrometry and analytical chemistry. We greatly appreciate the helpful manuscript reviews of R. A. Duncan and M. J. Defant.

\section{REFERENCES}

Aurelio, M. A., Rangin, C., Barrier, E., and Muller, C., 1990. Tectonique du segment central de la faille Philippine (Région de Bondoc-Masbate): un dérochement très récent. C. R. Acad. Sci. Ser. 2, 310:403-410.

Bellon, H., Quoc Buu, N., Chaumont, J., and Philippet, J. C., 1981. Implantation ionique d'argon dans une cible support: application au traéage isotopique de l'argon contenu dans les minéraux et les roches. C. R. Acad. Sci. Ser. 2, 292:977-980.

Cassignol, C., David, B., and Gillot, P. Y., 1977. Contribution au dosage de l'argon dans l'échantillon de glauconite Gl-O. Geostand. Newsl., 1:105-106.

Cox, A., and Dalrymple, G. B., 1967. Statistical analysis of geomagnetic reversal data and the precision of potassium-argon dating. $J$. Geophys. Res., 72:2603-2614.
Gervaiso, F. C., 1971. Geotectonic developments of the Philippines. J. Geol. Soc. Philipp., 25:18-38.

Gill, J. B., 1981. Orogenic Andesites and Plate Tectonics: New York (Springer-Verlag).

Kudrass, H. R., Heidicke, M., Cepek, P., Kreuzer, H., and Müller, P., 1986. Mesozoic and Cenozoic rocks dredged from the South China Sea (Reed Bank area) and Sulu Sea, and their significance for plate tectonic reconstructions. Mar. Pet. Geol., 3:19-30.

Kudrass, H. R., Müller, P., Kreuzer, H., and Weiss, W., 1990. Volcanic rocks and tertiary carbonates dredged from the Cagayan Ridge and the Southwest Sulu Sea, Philippines. In Rangin, C. Silver, E. A., von Breymann, M. T., et al., Proc. ODP, Init. Repts., 124: College Station, TX (Ocean Drilling Program), 93100.

Mahood, G. A., and Drake, R. E., 1982. K-Ar dating young rhyolitic rocks: a case study of the Sierra La Primavera, Jalisco, Mexico. Geol. Soc. Am. Bull., 93:1232-1241.

Marchadier, Y., 1988. La terminaison de la fosse de Manille en domaine continental. Etude stratigraphique et tectonique des îles de Mindoro-Tablas (Philippines) [thesis]. Univ. of Paris VI.

Marchadier, Y., and Rangin, C., 1989. Passage subduction-collision et tectoniques superposées à l'extrémité méridionale de la fosse de Manille (Mindoro - Tablas, Philippines). C. R. Acad. Sci. Ser. 2, 308:1715-1720.

Maury, R. C., 1984. Les conséquences volcaniques de la subduction. Bull. Soc. Geol. Fr., 26:489-500.

Moore, G. F., and Silver E. A., 1982. Collision processes in the Northern Molucca Sea. In Te Tectonic and Evolution of Southeast Asian Seas and Islands (Pt. 2). Geophys. Monogr. Ser., 27:360372.

Morrice, M. G., Jezek, P. A., Gill, J. B., Whitford, D. G., and Monoarfa, M., 1983. An introduction to the Sangihe arc: volcanism accompanying arc-arc collision in the Molucca Sea, Indonesia. J. Volcanol. Geotherm. Res., 19:135-165.

Peccerillo, A., and Taylor, S. R., 1976. Geochemistry of Eocene calc-alkaline volcanic rocks from the Kastamonu area, northern Turkey. Contrib. Mineral. Petrol., 58:63-81.

Rangin, C., Jolivet, L., Pubellier, M., and Tethys Pacific Working Group, 1990a. A simple model for the tectonic evolution of Southeast Asia and Indonesia regions for the past $43 \mathrm{Ma}$. Bull. Soc. Geol. Fr., 6:887-905.

Rangin, C., Bellon, H., Benard, F., Letouzey, J., Müller, C., and Sanudin, T., 1990b. Neogene arc-continent collision in Sabah, Northern Borneo (Malaysia). In Angelier, J. (Ed.), Geodynamic Evolution of the Eurasian Margin. Tectonophysics, 183:305-319.

Rangin, C., Pubellier, M., 1990. Subduction and accretion of oceanic fragments along the Eurasian margin: southern Japan-Philippine region. Some constraints for continental growth. In Aubouin, J., and Bourgois, J. (Eds.), Tectonics of Circum Pacific Continental Margins, (V.S.P International Publ.), 139-144.

Rangin, C., Silver, E. A., von Breymann, M. T., et al., 1990. Proc. ODP, Init. Repts., 124: College Station, TX (Ocean Drilling Program).

Rangin, C., Stephan, J. F., Butterlin, J., Bellon, H., Müller, C., Chorowicz, J., Baladad, D., in press. Collision néogène d'arcs volcaniques dans le centre des Philippines. Stratigraphie et structure de la chîne d'Antique: île de Panay. Bull. Soc. Geol. Fr. Santa Cruz, J. R., Letargo, M.R.R., and Samaniego, C. L., 1989. Petrography and chemistry of high $\mathrm{Mg}$, biotite-rich pillow-lavas from Southwestern Panay, Philippines. Tectonophysics, 168:137149

Silver, E. A., McCaffrey, R., Smith, R. B., 1983. Collision, rotation, and the initiation of subduction in the evolution of Sulawesi, Indonesia. J. Geophys. Res., 88:9407-9418.

Steiger, R. H., and Jäger, E., 1977. Subcommission on geochronology: convention on the use of decay constants in geo- and cosmochronology. Earth Planet. Sci. Lett., 26:359-362.

Sun, S.-S., Nesbitt, R. W., and Sharaskin, A. Y., 1979. Geochemical characteristics of mid-ocean ridge basalts. Earth Planet. Sci. Lett., 44:119-138.

Vogt, E. T., and Flower, F. J., 1989. Genesis of the Kinabalu (Sabah) granitoid at a subduction-collision junction. Contrib. Mineral. Petrol., 103:493-509. 
Wakita, H., Rey, P., and Schmitt, R. A., 1971. Abundances of the 14 rare-earth elements and 12 other trace-elements in Apollo 12 samples: five igneous and one breccia rocks and four soils. Proc. 2nd Lunar Sci. Conf., 1319-1329.

Walther, H. W., Förster, H., Harre, W., Kreuzer, H., Lenz, H., Müller, P., and Raschka, H., 1981. Early Cretaceous porphyry copper mineralization on Cebu Island, Philippines dated with $\mathrm{K}-\mathrm{Ar}$ and Rb-Sr methods. Geol. Jahrb., D48:21-35.
Wolfe, J. A., 1981. Philippine geochronology. J. Soc. Geol. Phil., 35:1-30.

Date of initial receipt: 3 July 1990

Date of acceptance: 28 February 1991

Ms 124B-163 